\title{
Conditions d'apparition et formes de rebond d'un outil de forage tricône
}

\author{
C. Putot ${ }^{1}$ et C. Mabile 2 \\ 1 Institut français du pétrole, 1 et 4, avenue du Bois-Préau, 92852 Rueil-Malmaison Cedex - France 2 \\ Geoservices, 7, rue Newton, BP 20, 93151 Le Blanc-Mesnil - France e-mail: claude.putot@ifp.fr
}

\begin{abstract}
Résumé - Le mode de vibration longitudinal du train de tiges peut, dans certaines conditions de forage, dégénérer en rebond avec séparation périodique à l'interface outil tricône, roche ; il va de soi que ce comportement doit être évité.

En présence de roches compactes, on a coutume d'associer le rebond à la présence d'un motif à l'interface $r$ roche-outil, dit motif trilobé en raison de sa symétrie ternaire. Il se matérialise par une ondulation périodique, sensiblement sinusoïdale, du front d'abattage.

En roches « tendres », l'allure du phénomène de vibration longitudinale est plus « chaotique » et fait l'objet d'investigations spécifiques liant dynamique et expulsion de copeaux (programme Cutclean).

En roches «dures », l'expérience prouve que le motif est repris à chaque passage d'outil par érosion différenciée des ondulations, sans qu'il y ait nécessairement décollement. Il s'ensuit une rotation d'ensemble du motif d'interface, de l'ordre de quelques degrés par tour, liée au décalage de phase entre effort de coupe et ondulation.

Le phénomène de rebond se manifeste préférentiellement lorsqu'il y a accord entre la fréquence de défilement des cônes - tributaire de la vitesse de rotation - et une fréquence propre du système de forage. Mais le phénomène peut également se manifester loin des résonances prévues par l'analyse linéaire.

L'interprétation présentée dans cet article, issue du domaine d'étude de l'usinage des métaux, est une étude de type formulation linéarisée.

Le critère de stabilité définit, pour un système donné, l'effort limite comme produit d'un terme caractéris tique de la formation par la raideur dynamique du mode de réponse de la structure. Ce terme est analogue au coefficient d'Archard dans une loi d'usure. Le coefficient de proportionnalité entre effort normal et profondeur de passe n'a d'ailleurs (la confusion est fréquente) aucun lien avec le module d'élasticité, mais davantage avec la caractéristique de cohésion de la roche.

Ainsi, et conformément à l'intuition, le rebond se manifestera préférentiellement avec une roche très résistante et une structure souple pour la fréquence envisagée.

Des considérations d'efficacité de coupe, liées à la compatibilité plus ou moins grande de la géométrie du cône et de la courbure de l'interface du motif trilobé, sont dégagées et cadrent parfaitement avec une dis symétrie observée sur les enregistrements (Trafor) du couple entre maxima et minima ; les phases de décollement et de reprise de contact en cas de rebond sont également compatibles avec les extrema de poids sur outil.
\end{abstract}

Mots-clés : outil tricône, rebond de l'outil, motif trilobé, stabilité, forabilité dynamique, autoexcitation, régénération de motif.

\begin{abstract}
Conditions of Appearance and Forms of Lift-off Dynamics (Bit bouncing) for a Roller Cone Drill Bit - The longitudinal vibration mode of the drill string can, in certain drilling conditions, degenerate into bit-bouncing with periodic separation at the roller bit cone, rock interface; it goes without saying that this behavior must be avoided.

In the presence of compact rocks, we are used to associating the lift-off dynamics to the presence of a pattern at the rock-bit interface, known as a bottom hole pattern because of ils ternary symmetry. It emerges by a more or less sinusoidal, periodic undulation of the cutting edge. On "soft rocks", the speed of the longitudinal vibration phenomenon is more "chaotic" and is the subject of specific investigations relating dynamics and the expulsion of fragments (Cutclean programme).

On "hard" rocks, the experience proves that the pattern is repeated on each passage of the bit by differentiated erosion of the undulations, without there necessarily being detachment. A general rotation of the interface pattern then occurs, in the order of a few degrees per turn, related the phase shift between cutting effort and undulation.
\end{abstract}

The bounce phenomenon appears especially when there is agreement between the running frequency of the cones-dependent on the rotational speed-and a frequency which is unique to the 
drilling system. However the phenomenon can also appear a long way from the resonance predicted by the linear analysis.

The interpretation presented in this report, from the machining study field of the metals, is a linearised formulation type study.

The stability criteria defines, for a given system, the limit effort as a product of a term which is characteristic of the formation, similar to the Archard coefficient in a wear theory, by the dynamic rigidity of the response mode of the structure, for the frequency which is likely to be induced. The proportionality coefficient between normal force and depth of pass has, despite frequent confusion, no link with the elasticity module, but more with the cohesion characteristic of the rock. Thus, in compliance with intuition, the lift-off dynamics will preferentially appear with a very resistant rock and a flexible structure for the frequency considered.

Factors of cutting efficiency related to the more or less large compatibility of the geometry of the cone and the curvature of the interface of the bottom hole patterns are cleared and correspond perfectly with a dissymmetry observed on the recordings (Trafor) of the torque between maximum and minimum; the detachment and contact repeat phases in case of bit-bouncing are also compatible with the extreme weight on the bit.

Keywords: roller cone bit, bit-bouncing, lift-off dynamics, bottom hole pattern, lobes, stability analysis, cutting dynamics, self induced mechanism, regenerative effect.

\section{PRÉSENTATION GÉNÉRALE}

\subsection{Organisation de l'article}

Après une description rapide du comportement mécanique du système de forage et des préoccupations du foreur liées au fonctionnement en cours d'opération, on montre le lien entre l'aptitude au forage et le régime de vibrations, en nous restreignant au mouvement longitudinal (selon l'axe du puits).

Les bases d'un modèle emprunté au domaine de l'usinage sont évoquées. L'apport original de l'étude consiste plus précisément

- en la démonstration de la cohérence de théories partielles ou éparses puisque relevant d'intérêts, d'états

d'esprit et de domaines d'application différents ;

- en la vérification expérimentale, en cours d'opération, de la succession des phases prévues par les théories pour les diverses grandeurs mécaniques caractéristiques.

\subsection{Description succincte du système de forage}

Le forage en mode dit « rotary » (fig. 1) consiste à entraîner depuis la surface, un train de tiges transmettant à l'outil forage (trépan), situé au fond du puits, un effort longitudinal de compression en fonctionnement normal, dit « poids s outil », et un « couple». Une certaine énergie mécanique i dépensée en surface pour entretenir cette rotation et permet le travail de désagrégation de la roche par les taillants l'outil ; l'ensemble du dispositif est soutenu au niveau i derrick par un effort contrôlé dit « traction au crochet ».

Masse des tiges et frottements dans le puits conditionne en fait le «poids sur outil » de sorte que la connaissance de traction au crochet ne permet qu'une estimation imparfaite ce poids, particulièrement en puits incliné.

Un élément important du fonctionnement du système c le fluide de forage (boue) dont le rôle est multiple mais comprend l'évacuation des déblais ; pour simplifier, et en l'absence de couplages significatifs concernant un transfert entre énergie mécanique et énergie hydraulique, on estime que l'énergie des pompes est associée à la remontée des déblais et on évoque la puissance hydraulique à l'outil.

Les outils traditionnels d'abattage de la roche au fond du puits sont de type tricône (fig. 2a); ils travaillent essentiellement en «poinçonnant » la roche. Ce sont des outils relativement peu performants mais qui accommodent généralement les transitions de faciès sans réajustement de la commande du foreur. L'agressivité des tricônes se manifestant plutôt de manière longitudinale, les vibrations latérales sont réduites par rapport à celles subies, dans ce mode, par les outils mentionnés ci-après.

À l'inverse les outils PDC (fig. 2b), de conception plus récente, travaillent principalement - et lorsqu'ils sont en bon état - en cisaillement. Ce mode de rupture est plus efficace et favorise l'extraction naturelle des débris rocheux. Ils sont plus performants s'ils sont bien utilisés mais plus vulnérables lorsque la commande n'est pas adaptée. La structure des PDC est, soit très organisée en « lames » dont la géométrie hélicoïdale conditionne notamment l'évacuation des déblais, soit caractérisée par une implantation de taillants en apparence aléatoire, généralement destinée à briser des fréquences propres trop basses. 


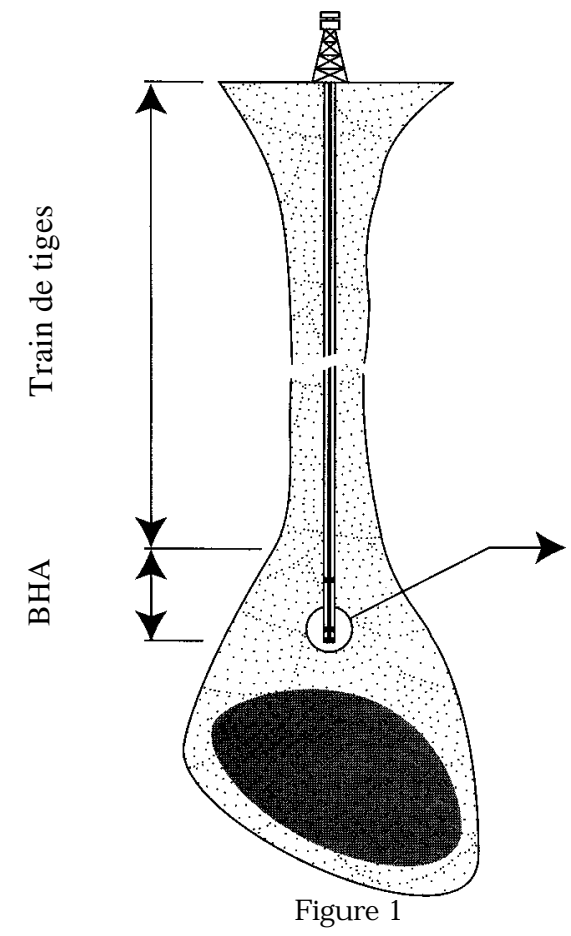

Le système de forage. The drilling system.

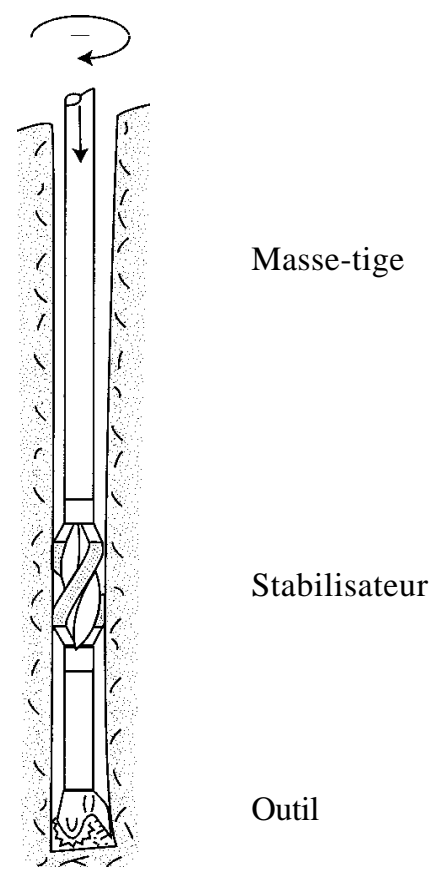




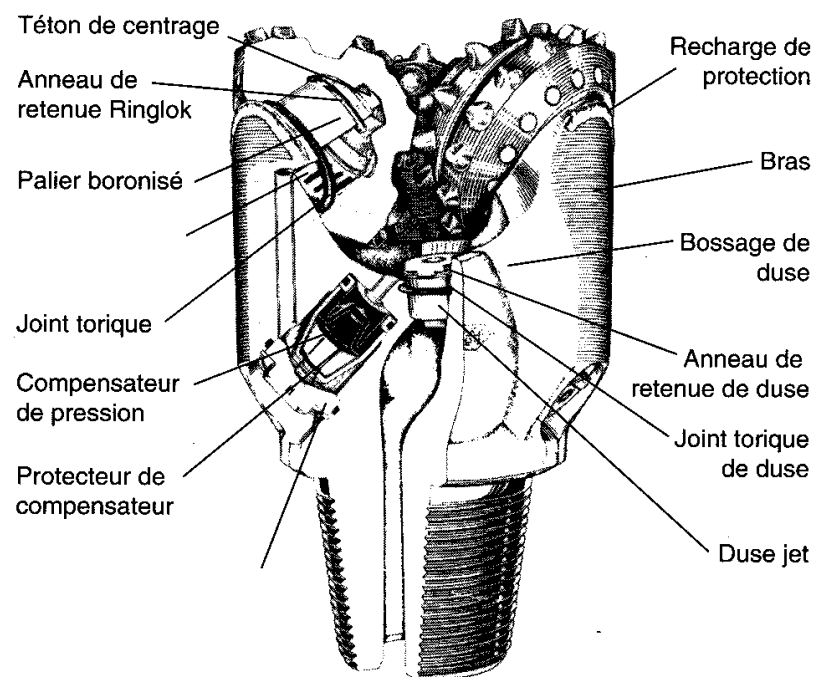

b

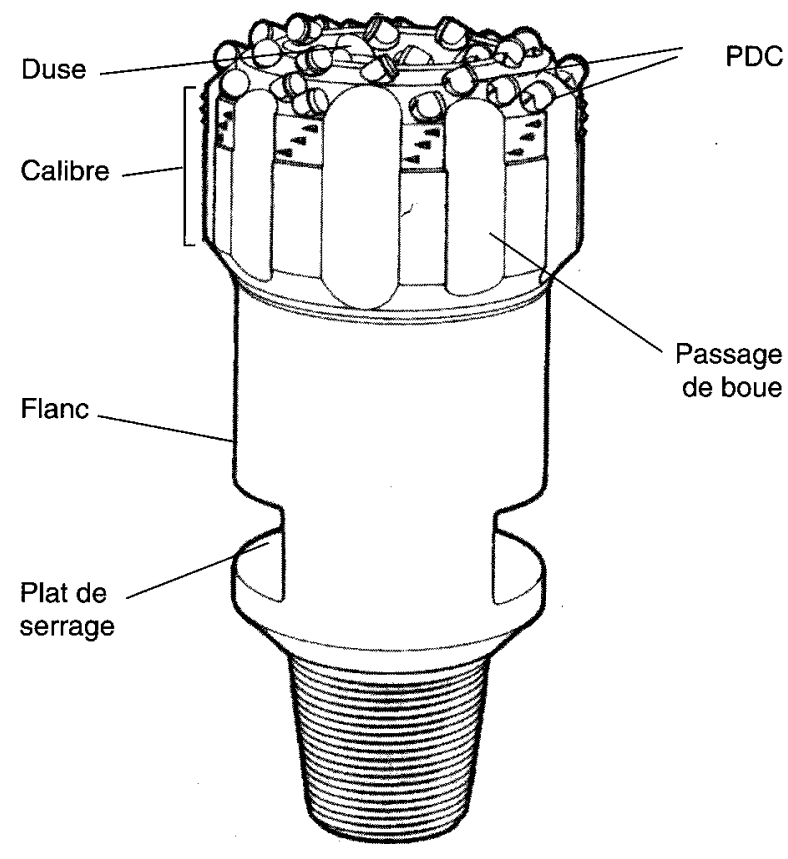

Bouchon de réservoir de lubrifiant

Source: d'après l'ouvrage de J.P. NGUYEN Le for Éditions Technip, 1

Figure 2

Exemples d'outils: tricône (a) et PDC (b). Examples of bits: roller cone (a) and PDC (b). 
Les outils précédemment décrits sont moins «typés » pratique : les cônes sont intentionnellement désaxés manière à introduire une composante cisaillement particulièrement utile en roches tendres (Winters, 1987) ; inversera( un outil PDC usé dont les taillants ont un méplat d'usure prononcé, poinçonnera la roche de manière totalement inefficace au lieu de la cisailler.

\subsection{Préoccupations du foreur}

Deux ensembles de préoccupations sont fréquemment rencontrés dans le domaine du forage. L'une concerne la limitation des vibrations, l'autre la performance de pénétration. Nous allons montrer qu'elles ne sont pas contradictoires.

\subsubsection{Vibration du train de tiges}

Un premier type de préoccupation concerne les vibrations du système de forage qui doivent rester dans des limites acceptables vis-à-vis des objectifs fixés. La limitation des vibrations revient à un compromis entre coût, qualité du puits foré et usure des équipements. En pratique, le foreur évite les dysfonctionnements vibratoires qui risquent d'occasionner des dommages importants au matériel et d'interrompre l'opération. Il réagit ainsi aux manifestations, en surface, du comportement vibratoire du système de forage.

Les spécialistes (Aldred, 1992) s'attachent à montrer le caractère parfois sournois du phénomène vibratoire, essentiellement localisé en profondeur, au voisinage de l'outil : les vibrations longitudinales et de torsion se transmettent bien du fond vers la surface, mais les vibrations de flexion, et particulièrement celles dites de précession, «passent mal » et ne peuvent être appréhendées, au mieux, que par l'intermédiaire de leur couplage avec les types de vibration non dispersifs.

\subsubsection{Forabilité traditionnelle}

Si l'on se trouve dans un régime de vibrations que l'on juge acceptable, on peut souhaiter améliorer la performance d'abattage (vitesse de pénétration). On entre alors plutôt dans les préoccupations du fabricant d'outil de forage dont le métier est de rechercher les bons profils d'outil et les meilleures technologies de taillant.

Le foreur assortit ses choix de recommandations sur la commande : poids sur outil, vitesse de rotation et débit hydraulique, en rapport avec le type de roche forée et, si possible, en fonction de l'usure des taillants, dans la mesure où cet état est « observable ».

Le rôle de la forabilité traditionnelle (Falconer, 1988 ; Detournay, 1992) est de fournir, en fonction de l'outil, des indicateurs calculés très simplement à partir de la commande (poids $\mathrm{W}$ vitesse de rotation $\mathrm{N}$ ) et de la réponse (vitesse de pénétration $\mathrm{R}$, couple $\mathrm{T}$ ). Ils contribuent à la caractérisation du type de roche forée et au suivi de l'usure des taillants. Ces indicateurs permettent également, dans une certaine mesure, de reconnaître si l'on s'écarte des conditions de forage ordinaires, soit que la roche traversée comporte des caractéris tiques à la rupture aberrantes (en tout cas différentes de celles de la formation précédemment évoquée), soit que l'on entre dans un régime de dysfonctionnement vibratoire. Les deux causes sont fréquemment associées (Putot, 1998).

\subsection{Vers une meilleure communication entre les deux domaines d'études}

\subsubsection{Forabilité en conditions dynamiques}

Une transition de faciès s'accompagne assez souvent, pour outil peu accommodant, de vibrations intenses, dues à u mauvaise adéquation de la commande aux nouvelles conditions de forage.

On saisit ainsi, à travers ces quelques commentaires, lien très étroit entre la recherche de la performance « fora lité » et la limitation des vibrations dont l'origine est essentiellement attribuée à la condition d'abattage de la roche 1 l'outil au niveau des taillants. Ce propos est à nuancer quelque peu, dans la mesure où l'on peut également imputer certaines vibrations à des conditions de contact particulièrement d'une partie de la garniture de forage en partie basse (BI ou bottom hole assembly) ou d'un stabilisateur (Abbassi; 1994).

Le caractère défavorable des vibrations quant aux perf, mances d'abattage ne fait aujourd'hui aucun doute chez spécialistes, même si, dans le passé, l'aspect «percussion pu être invoqué comme type de fonctionnement amélic envisageable. L'amélioration éventuelle en matière de performance de pénétration se fait généralement aux dépens de durée de vie de l'outil.

En ce qui concerne les vibrations plus classiques (subies passivement), des relations d'essais en laboratoire, ce d'AMOCO par exemple (Cooley, 1992 ; Sinor, 1992) coin] des relevés sur champ établis par fIFP récemment, ont $\mathrm{p}$ mis d'établir un lien indubitable entre la baisse de l'avance l'outil par tour, assimilable à une pénétration équivalence (depth of cut), et l'accroissement de l'intensité du régie vibratoire.

\subsubsection{Approches linéaires et non linéaires}


Les modèles mettant en œuvre de manière explicite la loi coupe comme moteur d'une dynamique vibratoire indésirable peuvent être classés en deux grandes catégories.

- Si l'on souhaite délimiter sommairement les condition (commande, paramètres caractéristiques) pour lesquels les vibrations ont tendance à s'amplifier au cours du terri on peut se satisfaire d'une analyse «linéarisée ». La théorie prévoit alors, dans un certain domaine de l'espace c paramètres ou de la commande, une croissance exponentielle de l'amplitude. On peut ranger dans ce catégorie les analyses de Dunayewsky (1985), soit sur modes séparés, soit sur les modes couplés. Les travaux Tobias (1961) et ceux de Tlusty (1986) sur l'usinage c métaux et les transpositions au forage (Zamudio, 19f 1988) appartiennent également à cette classe. Un exemple d'analyse linéarisée du système de forage est présenté Mabile (1996).

- Si l'on cherche à caractériser de manière plus quantitative les régimes de vibration que l'on ne souhaite pas nécessairement exclure, et à jauger dans ces conditions l'efficacité du processus d'abattage, il faut procéder à une analyse complète qui sera essentiellement non linéaire. En particulier, on s'intéressera à l'existence de régimes stationnaires avec apparition d'un cycle limite, ou à l'étude de transitoires. L'approche de Wu (1989), mettant en œuvre le comportement élasto-plastique du métal usiné, en régime dynamique, appartient à cette catégorie de méthodes.

Nous nous sommes attachés à ce dernier type de caractérisation lors d'une première approche du phénomène de rebond (Théron, 1996) et, plus récemment, à l'occasion d'un modèle de forabilité dynamique étendu aux conditions d'évacuation de déblais éventuellement défectueuses (Putot, 1997 ; Putot, Perreau et Constantinescu, 1998).

Avec ce dernier modèle, est organisé un cadre d'équations permettant de comptabiliser de manière synthétique les excitations et dissipations d'origine dynamique (coupe et frottement du méplat d'usure) et visqueuse (dégagement de débris rocheux mêlés à la boue, ceci sous fort gradient de pression). Ce modèle met bien en évidence le couplage fort entre variables d'état traduisant la concentration en débris au front de coupe et la dynamique du processus de forage. Autrement dit, le colmatage partiel au front d'abattage peut, dans certains cas, conditionner la dynamique de l'outil.

\subsection{Description du rebond de l'outil de forage}

Nous nous proposons dans cet article d'aborder un aspect du volet complémentaire de celui évoqué dans les publications (Putot, 1997 ; Putot, Perreau et Constantinescu, 1998) consacrées à l'excitation dynamique des outils en conditions hydrauliques défavorables, conditions pour lesquelles le processus normal de forage est entravé.

À l'inverse, il s'agit ici de caractériser un régime de forage en roches « dures », avec interaction mécanique très forte, susceptible de dégénérer en autoexcitation du système de forage.

Nous nous limitons, dans cet article, à l'excitation longitudinale du bas de la garniture de forage et rappelons en premier lieu quelques traits descriptifs et allusions bibliographiques.

\subsubsection{Visibilité en surface}

Le problème des oscillations longitudinales est, avec celui des variations de vitesse angulaire, celui qui se manifeste de la manière la plus spectaculaire en surface, puisqu'il autorise le foreur à affirmer que l'outil « rebondit au fond du trou ».

\subsubsection{Comportement longitudinal du tricône ; il justifie également l'aptitude au colmatage}

Le phénomène de rebond intervient préférentiellement avec un outil de type tricône dont l'énergie se développe plutôt dans le sens longitudinal (activité de poinçonnement en principe prédominante face à l'activité cisaillement) alors celle du PDC se manifeste plutôt dans le sens circonférentiel et transversal. Dans ce dernier cas, il y a génération de vibrations latérales de type précession et oscillations de torsion (cycles adhérence glissement ou stick-slip). Cependant, les oscillations de torsion existent également pour le tricône.

\subsubsection{Physionomie des enregistrements Trafor lors d'un rebond, selon le type de roche}

Le rebond se produit plus fréquemment dans les roches dures que dans les roches tendres, mais il n'est pas exclu dans ces dernières, où il semble lié à des problèmes d'expulsion de copeaux en raison d'un poids et/ou d'une vitesse excessif ou d'une hydraulique insuffisante. L'outil d'acquisition de données au fond Trafor permet d'enregistrer l'effort longitudinal au voisinage de l'outil. Le rebond implique une séparation outil-formation qui se traduit immédiatement par l'annulation du poids sur outil pendant une fraction du «cycle » qui peut être supérieure à la moitié de la durée de celui-ci. On notera que la physionomie du cycle en roche dure est plus pure et plus répétitive que celle du cycle en roche tendre (fig. 3). 
En roches dures, on a coutume d'associer le rebond à la présence d'un motif à l'interface roche-outil dit motif trilobé en raison de sa symétrie ternaire, qui se traduit par une ondulation périodique, sensiblement sinusoïdale, du front de taille (fig. 4).

\subsection{5 Évolution permanente du motif par érosion ; impression globale de rotation à vitesse constant du motif}

L'expérience, conduite avec Trafor par exemple, prouve que le motif est repris à chaque rotation de l'outil par érosion différenciée des ondulations sans qu'il y ait d'ailleurs nécessairement décollement de l'outil (absence de rebond). Ainsi, on a pu constater (fig. 5) une rotation du motif d'interface de quelques degrés par tour liée, comme on le verra plus lois au décalage de phase entre l'effort de coupe et l'ondulation. Les travaux de Pavone puis ceux de Mabile et Rey-Fabret (non publiés) ont mis expérimentalement en évidence cette rotation du motif. Parfois, le motif d'interface présente, non pas une symétrie ternaire, mais une symétrie d'ordre 6 ou 9. Autrement dit, entre deux cônes, on peut observer deux ondulations $(\mathrm{N}=1$ ou $\mathrm{N}=2)$ moins une fraction infime représentant érosion due au déphasage $\mathrm{E}$ (notations du chapitre 2.1).

\section{Rebond d'outil en roches tendres} (effet fluide)

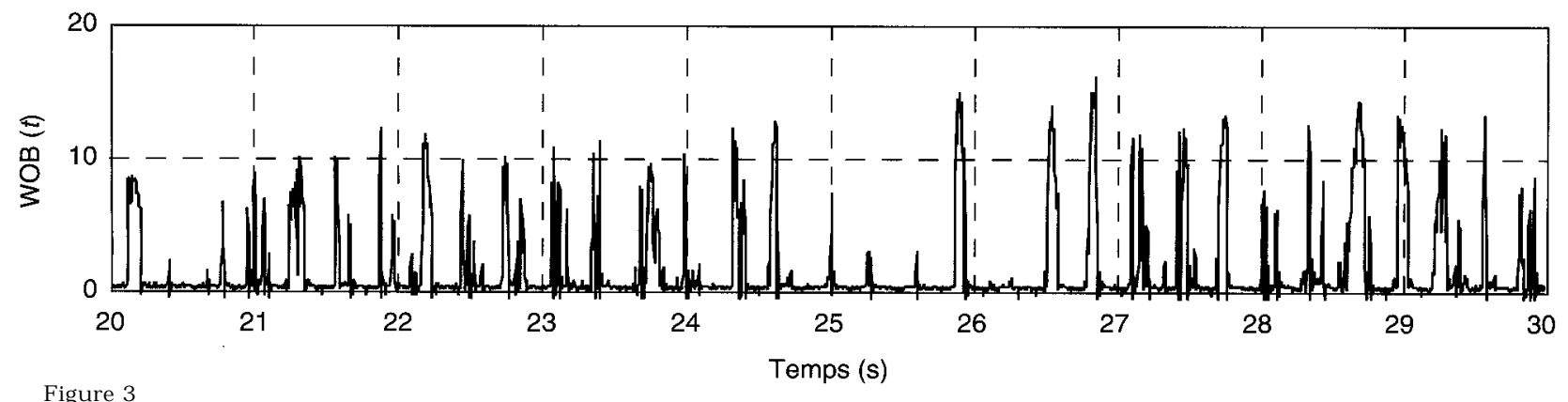

Deux exemples de dynamique longitudinale à l'outil d'après les données Trafor (d'après J.

Guesnon). Two exemples of longitudinal dynamics at the bit according to the Trafor

data (courtesy of J. Guesnon).
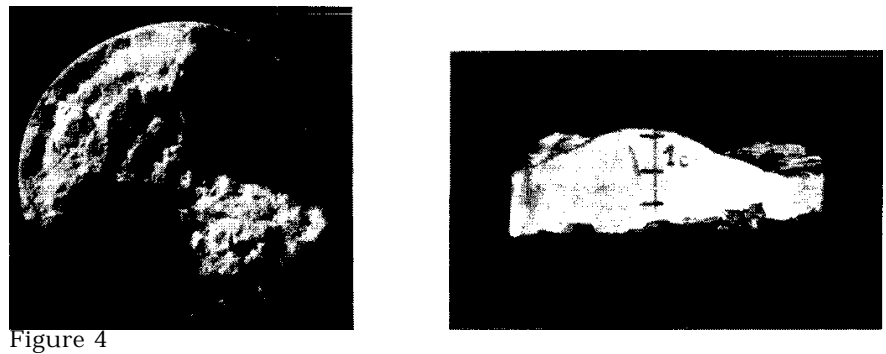

Représentation géométrique du trilobe (d'après Tag). Geometrical representation of the lobe (according to Tag).

1.5.6 Durée relative de la séquence de « vol » dans le cycle (I'analogue de la phase adhérence du stick-slip)

Les proportions de temps de « vol» (perte de contact de l'outil sur le trilobe se traduisant par une pénétration instantanée nulle) sont très variables d'un enregistrement à l'autre, et les simulations numériques reflètent cette diversité. Il s'agit de calculs par essence fortement non linéaires, avec rupture de contact et changement radical de conditions aux limites. On pourra se reporter aux travaux conduits au CEA en collaboration avec fIFP (Politopoulos, 1993 ; Ribes, 1993) (fig. 6a, 6b, 6c, 6d). Les analyses conduites plus récemment par Spanos (1994) comportent des phases de vol généralement plus courtes et dont le déclenchement est moins fréquent, la périodicité faible et d'apparence plutôt aléatoire. La diversité des physionomies de réponse est probablement à imputer à la « raideur de choc » prise pour simuler la loi d'interface en forabilité. Anticipant sur le contenu de la formulation plus réaliste développée au chapitre 2.1 , on note que ces analyses ne prennent pas en compte l'érosion du profil, de sorte que la dynamique est celle du mouvement d'outil avec contact unilatéral (mais possibilité de séparation), sur géométrie imposée mais invariable dans le temps. 
Il va sans dire que la durée de la phase de vol illustrée dans la figure 6a-6d est très exagérée par rapport à celle intervenant dans un processus plus réaliste conduisant à un trilobe.

a

Poids sur l'outil
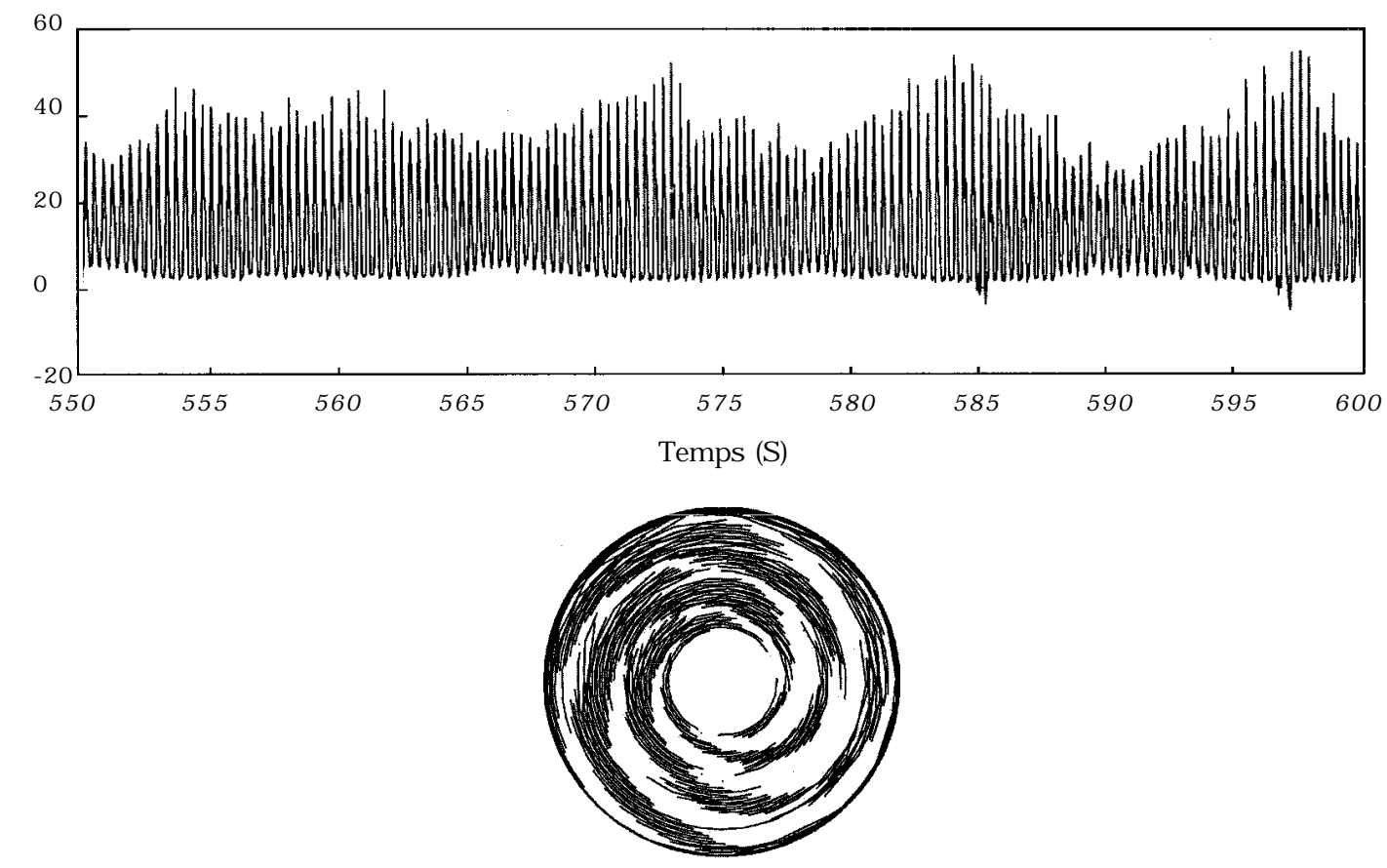

$\mathrm{b}$

\section{Poids sur l'outil}
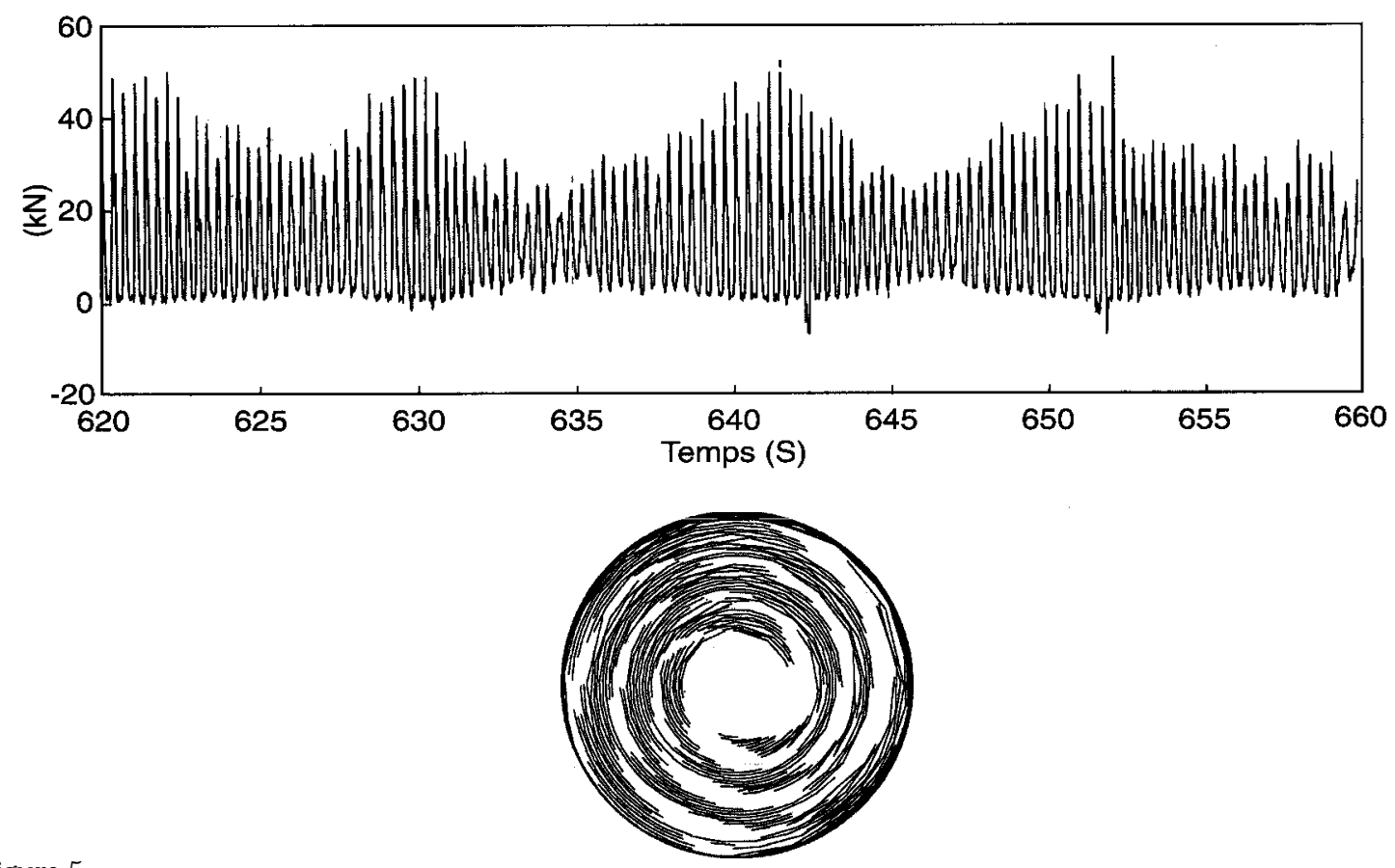

Figure 5

Mise en évidence de la rotation du motif trilobé en présence de rebond, à chaque révolution, visualisée d'après les enregistrements Trafor (d'après D. Pavone et 1. Rey Fabret).

Highlighting of the rotation of the bottom hole pattern in the présence of lift-off dynamics, on each revolution, seen according to the Trafor recordings (courtesy of D. Pavone and L Rey Fabret). 

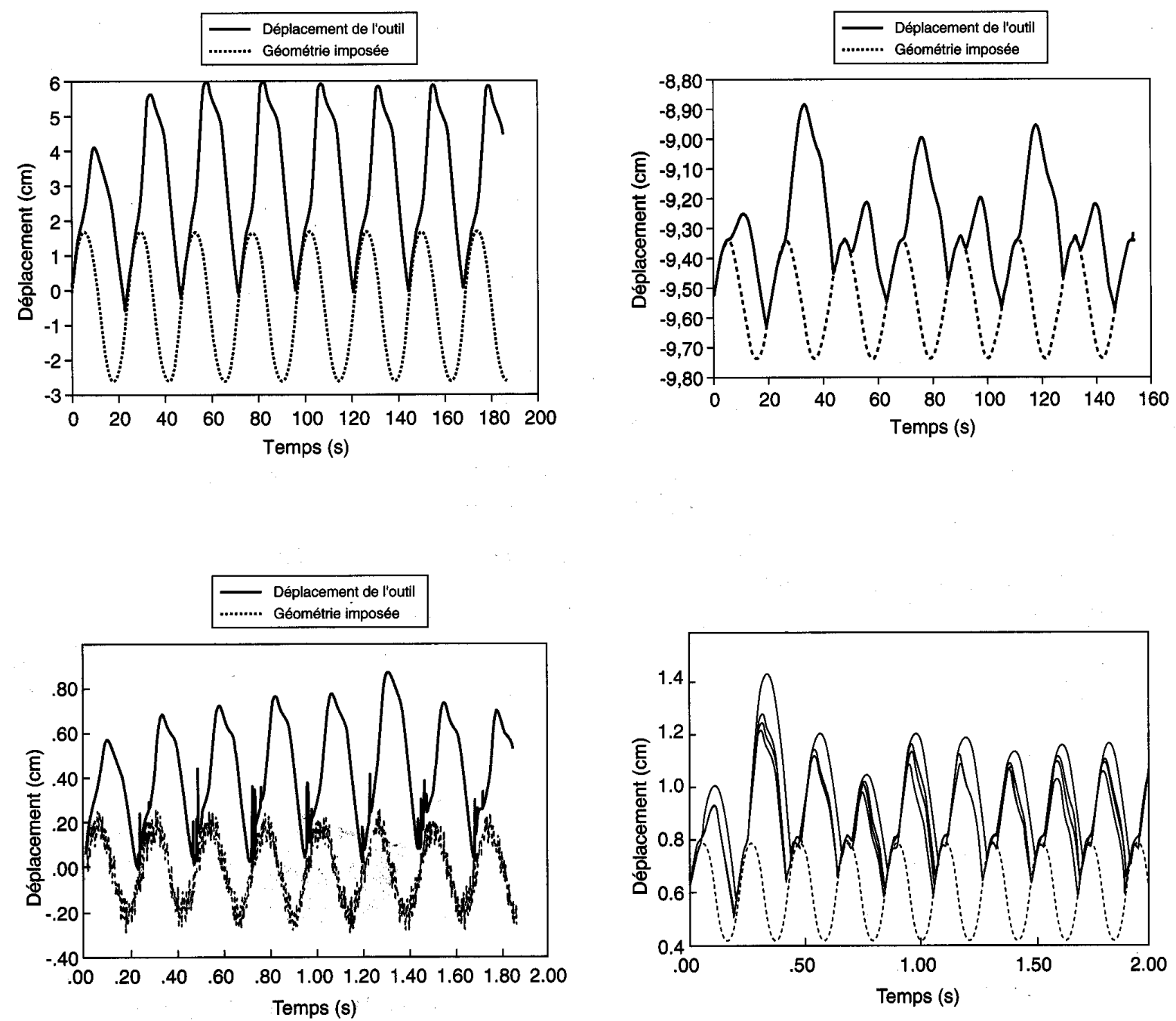

Figure 6

Analyse du rebond par décomposition sur base modale, avec le logiciel Castem 2000. Figures 6a, 6b, 6c : d'après Politopoulos (1993). Figure 6d : d'après Ribes (1993).

Analysis of the lift-off dynamics by analysis on modal base, with the Castem 2000 software. Figure 6a, 6b, 6c: according to Politopoulos (1993). Figure 6d: according to Ribes (1993).

\subsubsection{Manifestations du rebond et vibrations du train de tiges}

La fréquence du phénomène est conditionnée, comme on l'a vu, par la vitesse de rotation de l'outil et l'entretien simultané d'un régime d'oscillations longitudinales, puisqu'il y a reprise du motif trois fois par tour, à chaque passage de cône, au petit décalage de phase près, mentionné précédemment.

Il semblerait que le phénomène de rebond se manifeste préférentiellement lorsqu'il y a coïncidence entre la fréquence de défilement des cônes en un point donné du massif (donc tributaire de la vitesse de rotation) et une fréquence propre, généralement du mode longitudinal des tiges, parfois mode de torsion du système de forage (Dareing, 1984 Spanos, 1994). En pratique, des fréquences de l'ordre de quelques Hz, correspondant aux vitesses de rotation usuelles; sont observées, lorsqu'il y a excitation du système. Spano (1994) constate fréquemment, dans ses résultat, le phénomène de rebond associé aux résonances, mais parfois également dans des conditions qui en sont fort éloignée ; les non linéarités en justifient l'existence.

\subsubsection{Amplitude du décollement}

L'amplitude de la séparation est, dans les conditions usuelles, de l'ordre de quelques millimètres et du même ordre de grandeur que l'amplitude du trilobe. Il est évidemment instructif de relier cette amplitude au temps de vol, connaissant l'ordre de grandeur des accélérations longitudinales relevées sur les enregistrements, en faisant usage de la relation: $y=W x$. 


\subsubsection{Oscillations longitudinales et rebond ; traitement linéarisé}

Des variations importantes de l'effort longitudinal peuvent se manifester sans qu'il y ait pour autant annulation de poids et donc rebond à proprement parler. C'est surtout l'analyse de ce genre de phénomène d'amplitude limitée qui a fait l'objet de travaux importants dans le passé, tant dans le domaine du forage que dans celui de l'usinage des métaux et c'est tout particulièrement dans ce dernier cadre que, chronologiquement, l'effort a porté (Tobias, 1961 ; Tlusty, 1986).

\subsubsection{Principe de l'analyse linéaire ; boucle de rétroaction}

Limites de l'étude

Les analyses de type linéaire sont fondées sur la description des conditions définissant le rafrâichissement de la trace lais sée par l'outil (on parle aussi de régénération de profil ou d'ondulation) avec discussion des conditions de compatibilité d'une loi d'interface et de la réaction du bâti.

De manière très succincte, le critère de stabilité revient à définir, pour un système donné, un effort limite à l'interface. Cet effort dépend d'un terme adimensionnel produit de la souplesse du sol (au sens de la forabilité, voir $\S 2.1 .2$ et 2.2 ) et de la raideur du mode de réponse de la structure, compte tenu de la fréquence caractéristique considérée (raideur dynamique). Au-dessous de ce seuil, le système évolue de manière stable.

\subsubsection{Conséquences sur les conditions d'apparition du rebond}

Autrement dit, et conformément à l'intuition, raideur en forabilité et raideur du système de forage interviennent sur le rebond de manière antagoniste : le rebond se manifestera préférentiellement, pour la fréquence envisagée, avec une roche fortement réactive et une structure souple.

\subsubsection{Schéma simplifié d'autoexcitation longitudinale de l'outil}

Théron (1996) a présenté une formulation analytique très simplifiée du problème, qui comporte certaines ressemblances intéressantes dans les résultats avec la présentation de Tlusty (1986).

\subsubsection{Conditions éventuelles de couplage des oscillations longitudinales et circonférentielles}

Pour ce qui est du couplage des oscillations longitudinales et de torsion, il ne semble pas qu'il y ait des cas où, simultanément, rebond et stick-slip coexistent. Par exemple, sur les données Trafor en roches dures, les cycles de rebond sont évidemment associés à des annulations simultanées du poids et du couple au cours de la phase de séparation, ce qui est normal ; on pourrait en déduire que des fluctuations importantes du couple engendrent des fluctuations notables de la vitesse de rotation, mais ce n'est pas toujours le cas, en raison probablement de la souplesse et de l'inertie du système.

\section{APPROCHES LINÉAIRES DU PROBLÈME DE REBOND: RAPPELS BIBLIOGRAPHIQUES ET DISCUSSION}

\subsection{Le problème de fraisage et sa transposition au forage d'aprèsTulsty}

\subsubsection{Généralités sur l'usinage}

Il s'agit là d'une méthode, à l'évidence, approximative, destinée à cerner les conditions de stabilité de l'outil. L'approximation réside dans le fait que, lors de la rupture du contact occasionnée par le rebond, la concordance de phase entre le mécanisme excitateur et le retour de la structure n'a plus lieu d'être puisque la chaîne de fautoexcitation est rompue. À l'inverse, une analyse non linéaire, telle que celle réalisée par le CEA, permet de définir plus précisément le processus d'autostabilisation qui résulte de la séparation, et, d'autre part, de caractériser un éventuel cycle limite, lorsqu'il existe. En revanche, comme cela a été mentionné plus haut, la régénération d'ondulation n'a pas été incluse dans l'étude.

Dans le cadre linéaire, Dareing, Tlusty et Zamudio $(1987,1988)$ ont réalisé une transposition au forage d'une solution au problème de fraisage (Tlusty, 1986). Une description de ce dernier problème nous paraît utile.

\section{Objectifs généraux des modèles d'usinage}

Le but des investigations dans le domaine de l'usinage est de fournir des théories (modèles) permettant de définir au mieux des paramètres matériau et des conditions de coupe telles la vitesse et la géométrie de l'outil de coupe.

Ces théories sont utiles pour caractériser l'aptitude à l'usinage, la consommation d'énergie et, pour le plus important, la durée de vie de l'outil, liée aux contraintes et à la température de l'interface.

Les tendances actuelles sont au très grand perfectionnement des outils de découpe et à l'incorporation d'un contrôle actif, ce qui rend plus pressant le besoin de théories bien fondées.

Malgré la variété des outils de coupe et la diversité des formes d'interface, le processus de séparation de matière est toujours à peu près le même, de sorte que la mécanique de base peut être envisagée en considérant le mécanisme de coupe comme procédant à partir d'une arête anguleuse unique. Il est également habituel de limiter son attention au cas relativement élémentaire d'usinage orthogonal pour lequel l'arête de coupe rectiligne est 
parallèle à l'interface actuellement usinée et normale à la direction de coupe. Si la profondeur de coupe est petite comparée à la largeur d'interaction, le processus peut être caractérisé en déformations planes, ce qui simplifie grandement les théories en plasticité pouvant être utilisées.

Une simplification usuelle consiste à envisager la formation d'un copeau continu sans fissuration ni bourrelets et à considérer le processus comme stationnaire.

La composante dynamique : généralités

L'étude de la dynamique du processus et la prévention des phénomènes d'autoexcitation constituent un sujet d'intérêt, tant sur le plan scientifique que sur celui des enjeux économiques.

Trois mécanismes non indépendants ont été reliés au processus d'autoexcitation.

- L'effet vitesse traduit l'autoexcitation induite par un effort de coupe décroissant avec l'augmentation de la vitesse.

- L'effet géométrique résultant de la régénération d'ondulation se traduit par un déphasage entre les ondes successives consécutif au mouvement de l'outil en interaction avec la matière usinée lors de la coupe ; il est dû physiquement à la dissymétrie entre les efforts moyens de coupe associés aux phases respectives de descente puis de montée de l'outil le long des flancs de l'ondulation. Il en résulte une modulation de l'épaisseur du copeau qui engendre et entretient une variation continue de l'effort de coupe et peut exciter la machine en particulier au voisinage des fréquences propres du bâti.

Manifestations de la stabilité et paramètres la conditionnant

Un mouvement relatif entre outil et pièce usinée conduit à des ondulations de l'interface qui peuvent être ou non entretenues au cours des passes successives. Un cas stable est celui pour lequel une vibration initiale accidentelle, due à une irré gularité locale par exemple, diminue dans les passes subséquentes ; au contraire, dans le cas instable, cette vibration s'amplifie exponentiellement dans le cadre d'une théorie linéaire. La limite de stabilité définit les conditions pour les quelles l'amplitude de la vibration demeure stationnaire.

Comme annoncé plus haut, la permanence des conditions d'autoexcitation dépend

- de la structure de la machine d'usinage et de la pièce usinée ;

- Des effets de couplage entre modes interviennent lorsque les fréquences propres des modes principaux sont proches la force de coupe agit dans une direction et peut provoquer une excitation dans une autre direction, créant des conditions de résonance.

- des caractéristiques en raideur et amortissement;

- de l'orientation des modes de vibration et des conditions de la coupe

- caractéristiques mécaniques du matériau usiné,

- épaisseur tranchée,

- vitesse de coupe,

largeur de copeau $b$; ce dernier paramètre est déterminant, l'étude de stabilité consiste justement à définir le seuil $b l t, n$ en dessous duquel il n'y a pas excitation et au dessus duquel l'excitation peut intervenir. Aux grandes largeurs de copeau, fautoexcitation intervient dans tous les cas.

Ces résultats s'interprètent immédiatement à l'examen de la figure 8.

\subsubsection{Modèle de représentation}

Du point de vue de la technique de calcul, Tlusty utilise, dans un premier temps, pour caractériser la fonction de transfert du bâti, un système linéaire à un degré de liberté et d'ordre 2 avec prise en compte d'une raideur et d'un amortissement pour le mode sélectionné au voisinage de la fréquence susceptible d'être excitée (approche ciblée dans un domaine de fréquences restreint).

L'équivalent de la loi de forabilité pour l'outil de forage s'énonce sous forme d'une loi de coupe où l'effort instantané est proportionnel à l'épaisseur actuelle du copeau, l'épaisseur tranchée correspondant à l'avance de l'outil par tour, l'épais seur $d u$ copeau est définie très précisément comme la différence entre la cote au tour précédent et la cote actuelle du taillant, supposant que l'outil se positionne immédiatement (hypothèse à remettre en question éventuellement, conformément à la discussion présentée par Théron en 1996).

La condition de stabilité de l'oscillation (non croissance ou non décroissance du copeau) est discutée très aisément par voie géométrique au moyen du diagramme de Nyquist (fig. 8a) associé à la fonction de transfert : 


$$
\begin{aligned}
G_{2, \xi}(s) & =\frac{n}{s^{2}+2 \xi \omega_{n} s+\omega_{n}^{2}} \\
r & =\frac{\omega}{\omega_{n}}
\end{aligned}
$$

où :

$r$ est le paramètre de la courbe dans la représentation de Nyquist où $s=-j \omega$;

$Z$ est l'amplitude complexe d'une vibration harmonique de pulsation $\omega$ et de fréquence $f$;

$Z_{0}$ est l'affixe correspondant à la révolution précédente, ou, dans le cas d'un outil périodique, à la période précédente;

$\omega_{n}$ est la pulsation propre.

La boucle de rétroaction' s'écrit très simplement en assurant la compatibilité dans le plan complexe des équations (1)

(2) et (3), soit

$$
F=b K_{\mathrm{S}}\left(Z_{\mathrm{O}}-Z\right)
$$

qui exprime la proportionnalité de l'effort $F$ à

- la profondeur tranchée actuelle Z074

- la longueur cumulée de coupe $b$

$$
Z=F G
$$

où $\mathrm{G}$ est la fonction de transfert du bâti (un degré de liberté, ordre 2).

Les équations (1) et (2) sont étudiées dans le cadre de l'hypothèse de stationnarité

$$
\text { IZI = IZOI }
$$

F désigne la partie fluctuante de l'effort de coupe dans la loi reliant les parties fluctuantes de l'effort et de l'épaisseur tranchée ;

$b=p \mathrm{~d} / 2$ est le coefficient de proportionnalité exprimant la dimension transversale cumulée du copeau ;

$\mathrm{K}_{\mathrm{s}} \quad$ est un réel positif reliant l'effort d'interaction à l'épaisseur du copeau, par unité de longueur de coupe (homogène donc à une pression) ;

$\mathrm{p} \quad$ désigne l'ordre de multiplicité ( $\mathrm{p}=3$ pour la transposition à un tricône) ;

$d \quad$ est le diamètre d'outil ;

$\mathrm{Z} \quad$ est la cote instantanée actuelle du copeau (t) ;

$\mathrm{Z}_{\mathrm{o}} \quad$ est la cote à la révolution précédente $\left(\mathrm{t}^{-} \mathrm{c}\right)$;

G est la fonction de transfert de la structure supposée à un degré de liberté et d'ordre 2

$$
G(r)=\frac{Z(r)}{F(r)}=\frac{u / k}{1-2 j \xi r-r^{2}}
$$

Elle est représentée dans le plan complexe de la figure 8a en normalisant l'effort à $F=1$, compte tenu d'un amortissement de 0,04 .

? (D est le retard de phase de $\mathrm{Z}$ sur la partie variable de l'effort de coupe $F$;

$E$ c est le décalage entre $Z$ et $Z_{o}$; c'est lui qui détermine la vitesse de rotation du motif d'ondulation ; plus cette valeur est faible (modulo $2 n$ ) et plus cette rotation et l'érosion qui lui est liée sont modérées ;

est la fréquence d'autoexcitation, c'est-à-dire celle du mouvement effectif ;

(1) La loi de comportement de la structure est supposée caractérisée par G, et $Z$ est la variable associée ; la forabilité est récapitulée par $\mathrm{K}_{\mathrm{s}}$ et la variable associée est Z-Z..; l'effort $\mathrm{F}$ est la grandeur d'interface permettant d'établir le lien. La solution du système et son évolution peuvent être obtenues par la voie des équations différentielles (Mazelet, 1995), mais ici, seule la condition de stationnarité nous intéresse et elle est soluble par de simples considérations de géométrie.

$\mathrm{f}_{\mathrm{n}} \quad$ est la fréquence propre du bâti associée à la fonction de $\operatorname{transfert~} G$;

$k \quad$ est la raideur statique pour le mode sélectionné ;

$\mathrm{u} \quad$ est un facteur d'orientation lié à l'écart entre la ligne d'effort $F$ et la direction de vibration $\mathrm{Z}$;

? $\quad$ est l'amortissement (la figure 7 a correspond à un amortis sement de 0,04). 
A) $N=2$

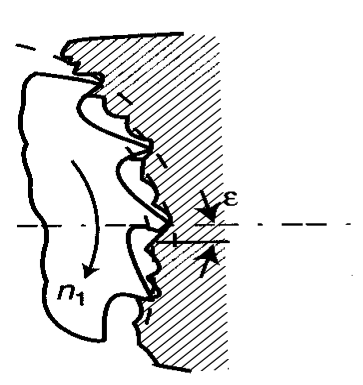

B) $N=6$

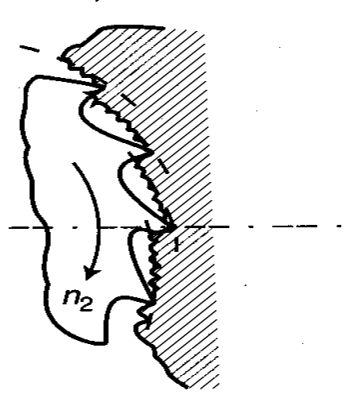

Figure 7
C) $N=1$

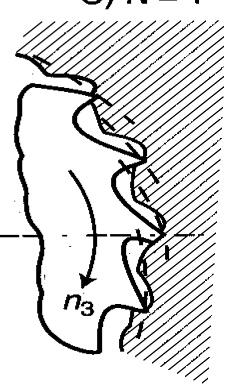

Modes de régénération d'ondulation (en fraisage) selon la vitesse de défilement de l'outil.

Regeneration of waviness (during milling) depending on the spindle speed.

L'objectif est de rechercher la valeur $d_{i u n}$ de diamètre d'outil correspondant à la limite de stabilité (limite de non croissance ou décroissance des oscillations Z).

Tenant compte de :

$$
Z_{o}-Z=2 R e[G(c o)]
$$

aisément déduit de (2) en conditions de stationnarité (3) et moyennant la loi de forabilité (1), le résultat s'exprime assez simplement sous la forme suivante (où l'on a particularisé à $p=3$, compte tenu de l'application tricône)

$$
\operatorname{diim}=3 \stackrel{1}{K s} \operatorname{Re}[G(c o)]
$$

Cette forme montre bien que l'effort tolérable, à la limite de stabilité, est d'autant plus important que le produit de termes figurant au dénominateur est plus petit, ce qui correspond, soit à une formation friable ( $K_{\mathrm{S}}$ petit), soit à une faible flexibilité dynamique (grande raideur) du système de forage ( $\operatorname{Re}[G(c o)]$ petit), soit, éventuellement, aux deux circonstances simultanées. L'échelle du forage stable résulte donc de la juxtaposition d'un élément " formation » $\left(K_{\mathrm{S}}\right)$ et d'un élément «structure » $(\mathrm{G})$.

a

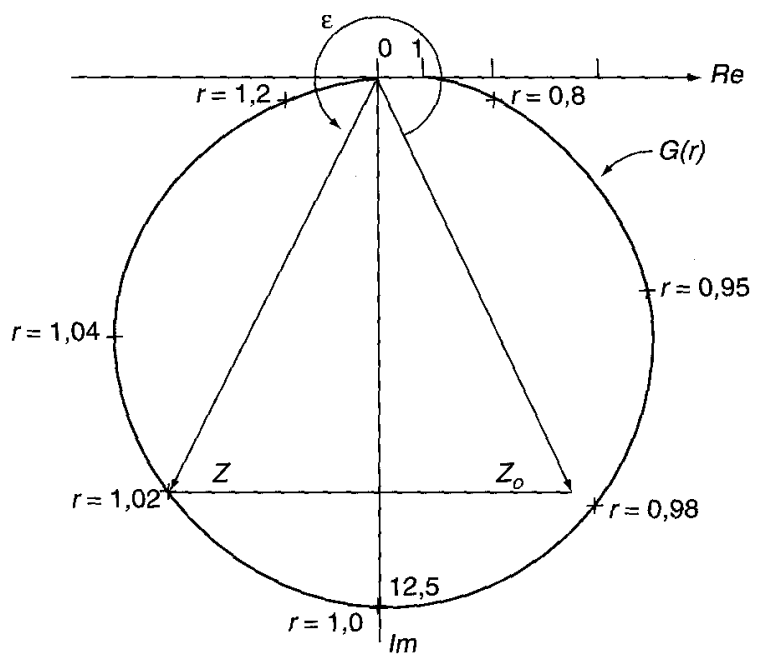

Figure 8a

Caractéristique de la fonction de transfert reliant la composante fluctuante de l'effort de coupe à la cote de l'outil (d'après Tlusty).

Characteristic of the transfer function linking the fluctuating component of the cutting force to the value of the bit laccording to Tlusty). 


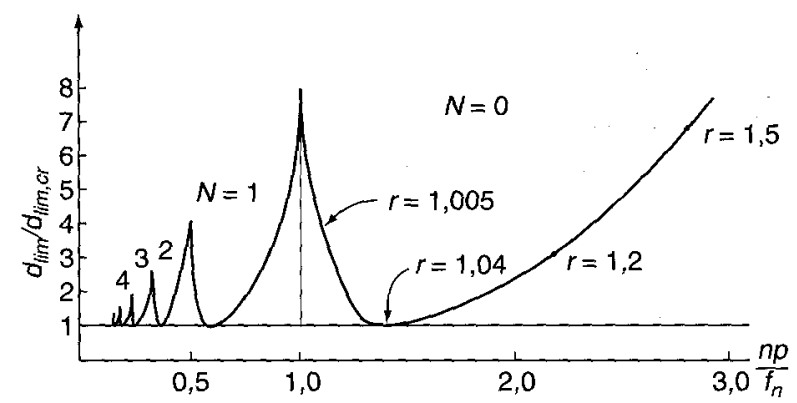

Figure $8 \mathrm{~b}$

Représentation de la limite de stabilité : effort maximal en fonction de la vitesse de défilement (d'après Tlusty). Representation of the stability limit: maximum force according to the running speed (according to Tlusty).

Un sous-produit intéressant de l'analyse, lié au déphasage :

$$
c=\arg Z_{o}-\arg Z
$$

entre la vibration du taillant (du cône, dans le cadre de transposition à l'outil tricône) à deux instants séparés d'une période de rotation de l'outil, est l'égalité suivante

$$
N+\$=f^{\prime \prime} 2 n n p
$$

Elle indique le nombre entier $N$ d'ondulations existant entre deux taillants (ou cônes) successifs (p ordre de multiplicité des taillants ; pour un tricône $\mathrm{p}=3$ ) ; $\mathrm{f}_{\mathrm{c}}$ est la fréquence d'autoexcitation ; les vitesses importantes $n$ sont caractérisées par un nombre $N$ peu élevé, éventuellement $N=0$, tandis que les vitesses faibles comportent un nombre important d'ondulations entre deux taillants successifs (fig. 7). Le train de tiges oscille pendant plusieurs périodes entre deux passages successifs de cônes en un point donné de la formation.

Dans la transposition au forage, on observera le plus souvent $N=0$ (trilobe) et parfois $N=1$ ou 2 (motifs d'ordre 6 ou 9 constatés par Rey-Fabret et Pavone et également mentionnés dans la littérature).

Le contour du domaine de stabilité dans le plan comportant en abscisses les vitesses réduites et en ordonnées les efforts est un « diagramme à lobes » (fig. 8b) évoquant celui représentatif de l'équation de Mathieu.

\subsubsection{Compléments : l'équation de Mathieu}

Le prototype le plus typique de l'instabilité paramétrique est celui correspondant au modèle de poutre droite rotulée-rotulée en flexion soumise à un chargement de traction compression harmonique.

On peut représenter le système au voisinage du premier mode par l'équation

$$
\frac{\mathrm{d}^{2} y}{\mathrm{~d} t^{2}}+2 \varepsilon \omega_{0} \frac{\mathrm{d} y}{\mathrm{~d} t}+\omega_{0}^{2}(1-2 \mu \cos \omega t) y=0
$$

\footnotetext{
$\mathrm{cA}_{\mathrm{o}} \quad$ est la pulsation propre du mode en l'absence du chargement;

E est l'amortissement réduit ;

$w \quad$ est la pulsation imposée par le chargement ;

g est l'amplitude fixant l'intensité du chargement oscillant.
}

Selon le positionnement de la fréquence d'excitation par rapport à la fréquence propre du système, il peut être plus difficile ou plus facile, selon le cas, de faire « flamber la poutre en dynamique ». Cette description 
qualitative assez intuitive est également assez évocatrice et représentative des oscillations longitudinales du système de forage.

Si $E=0$, l'équation se ramène à une équation de Mathieu. On sait que pour l'équation de Mathieu, il est facile de déstabiliser le système si la fréquence d'excitation est voisine de la fréquence propre ou de certains multiples et sousmultiples, mais la valeur de l'amortissement est un élément important conditionnant le résultat. La représentation du domaine de stabilité (fig. 9) évoque évidemment le diagramme à lobes de la théorie présentée par Tlusty.

La présentation de Dunayewsky (1985) des instabilités de précession résultant de fluctuations du poids est également d'inspiration très voisine.

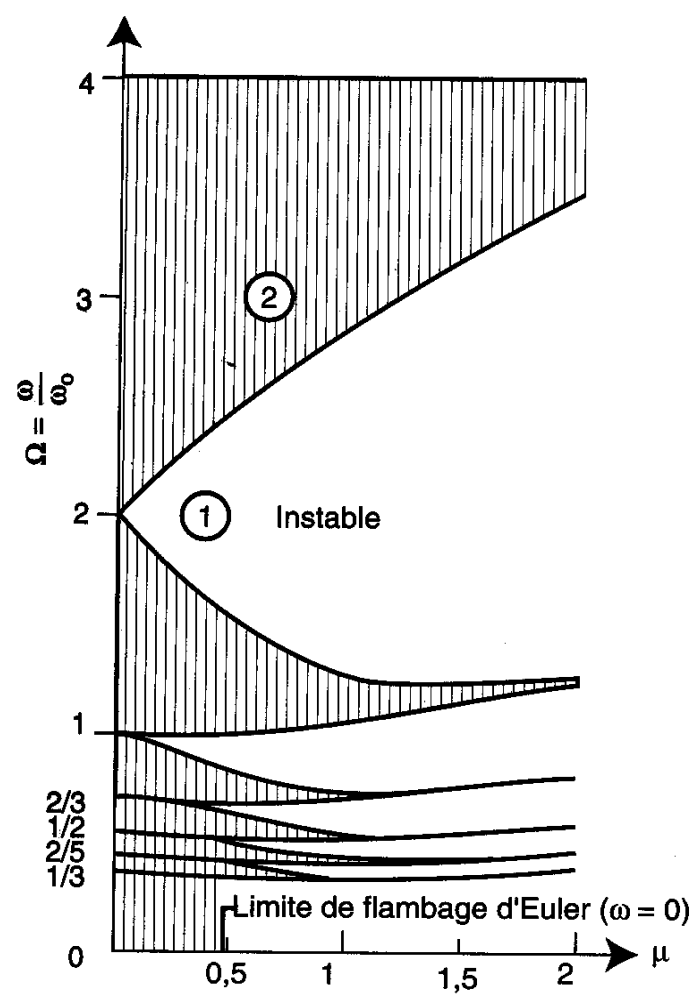

Figure 9

$\mathrm{Z}(\mathrm{t})-\mathrm{Z}(\mathrm{t}-1 r)=K_{u} F_{N}$

Exemple d'instabilité paramétrique : équation de Mathieu (sans amortissement); représentation de l'équation générale avec amortissement e. Example of parametric instability: Mathieu Equation (without damping); representation of the general Equation with $\mathrm{e}$ damping.

\subsubsection{Perfectionnements du modèle}

Dans le domaine de l'usinage, Tlusty envisage

- la participation simultanée de modes géométriquement orthogonaux ;

- l'influence de l'amortissement lié à la présence d'un méplat d'usure sur l'équivalent de la loi de forabilité ;

- la prise en compte de modes réels du bâti et notamment la proximité mutuelle de certains modes.

Par ailleurs, l'accent est également mis sur la fonction de transfert réaliste du système de forage qui s'éloigne notablement d'un système à 1 degré de liberté d'ordre 2.

Pour les faibles vitesses de rotation, un calcul détaillé (Tlusty, 1987) avec une fonction de transfert réaliste du système de forage conduit au résultat suivant : l'enveloppe des lobes de stabilité n'autorise, aux faibles vitesses, que des fluctuations d'effort vertical très petites ; ce résultat est à mettre en parallèle avec celui obtenu par Dunayewsky (1995) pour les oscillations de torsion. En outre, des amortisseurs spécifiques, s'ils sont bien dimensionnés, peuvent notablement réduire l'effort dynamique. 


\subsection{Approche analytique par le modèle spécifique de Théron}

Les travaux de Théron (1996) effectués dans un contexte analytique assez différent du précédent confortent les déductions de Tlusty (1986) et fournissent un cadre simplifié commode et synthétique. La présentation des résultats est illustrée dans la figure 10, reproduite de la thèse de Théron(1996), où l'on voit le rôle joué en abscisses par le produit $\mathrm{K}_{B} K_{u}: K_{B}$ est la raideur de structure. $K_{u}$, homogène à l'inverse d'une raideur, s'interprète comme coefficient d'une loi de forabilité assimilée à une loi d'abrasion, dite d'Archard. Cette loi exprime que le volume de matière produite est proportionnel à l'effort normal (et à la longueur parcourue par l'outil d'usure ou de coupe). Ce coefficient est à l'inverse de la puissance nécessaire à l'action de l'outil pour désagréger l'unité de volume de roche. C'est l'énergie spécifique de la mécanique des roches. On écrit :

$$
\mathbf{Z}(\mathbf{t})-\mathbf{Z}(\mathbf{t}-\mathbf{?})=\mathbf{K}_{\mathbf{u}} \mathbf{F}_{\mathbf{n}}
$$

$K_{u}$ élevé : fort dégagement de matière (roche friable) ; $K_{B}$ élevé : structure raide et corrélativement $R e(G)$ petit. La signification du produit de facteurs $K_{B} K_{u}$ est évidemment la même que celle du produit $3 K_{S} \operatorname{Re}[G(c o)]$ mis en évidence par Tlusty.

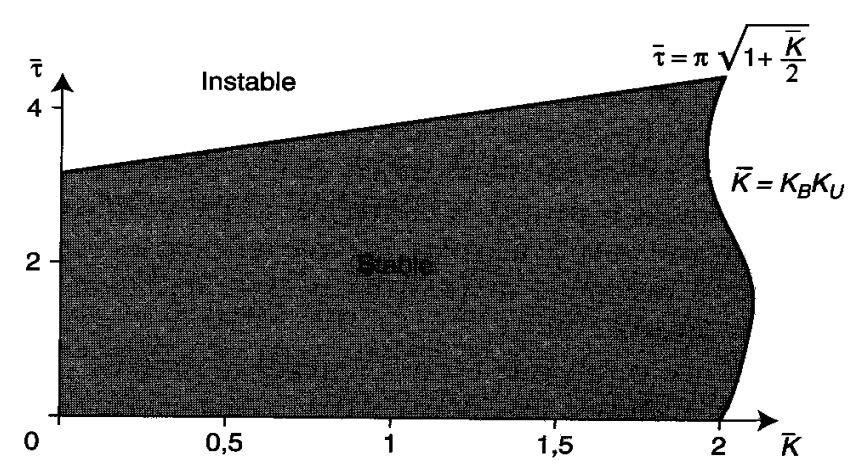

Figure 10

Limite de stabilité du modèle d'excitation longitudinale (Théron, 1996).

Stability limit of the axial vibration model (Théron, 1996).

Théron (1996) représente en ordonnée (fig. 10) une période adimensionnelle (période d'excitation corrigée d'un effet de structure).

On constate la concordance qualitative des déductions de Tlusty et Théron : zone de stabilité plus restreinte aux faibles vitesses (ces faibles vitesses correspondent à des ordonnées importantes dans le système de coordonnées choisi par Théron), avec domaine de stabilité d'autant plus étendu que le produit $K_{B} K_{u}$ est plus grand.

Quantitativement, dans la théorie de Tlusty comme dans celle de Théron, une confrontation avec des résultats sur champ nécessite de fixer certains ordres de grandeur ; c'est ce à quoi nous nous attachons maintenant en précisant numériquement les grandeurs $K_{B}$ et $K_{u}$.

\subsection{Application et discussion}

\subsubsection{Lien effort normal-épaisseur du copeau (Kd}

L'optique « aptitude au forage » des roches

Le lien entre effort normal (poids sur outil) et profondeur de passe 8, que l'on assimile à l'épaisseur de copeau, peut évidemment être fait indépendamment de la théorie d'Archard qui n'a, a priori, aucune raison d'être la loi adéquate pour le problème spécifique de la forabilité.

Aussi, les spécialistes s'attachent-ils à définir, tout d'abord expérimentalement, et sans a priori, les courbes représentatives de l'avance 8 en fonction du poids ; Sandia (Glowka, 1985) a rassemblé, pour l'outil PDC, un nombre considérable de données expérimentales qui ont été organisées et interpré tées par Detournay et Defoumy (1992). L'allure des résultats vaut également, dans une certaine mesure, pour les outils tricônes mais les résultats spécifiques récents pour ce type d'outil font défaut.

Allure des caractéristiques : poids, pénétration 
Les résultats se présentent de la manière suivante (fig. 11). La courbe liant 8 à W présente, aux faibles poids, un effet de seuil plus ou moins marqué selon les roches. En deçà de ce seuil, l'outil fonctionne inefficacement par un mécanisme s'apparentant à l'abrasion plutôt qu'à la coupe ; au delà, et tant que l'hydraulique le permet, la profondeur de passe est sensiblement proportionnelle au poids, la pente reflétant les caractéristiques à la rupture de la roche et, dans une moindre mesure, le concept d'outil.

On sait que cette pente est très importante pour les grès poreux (faible énergie spécifique) et il est notoirement connu que le contrôle du forage de ce type de roche est très difficile à garder, le fonctionnement étant, si l'on n'y prend pas garde, soit dans le domaine inefficace de l'abrasion, soit dans le domaine du bourragecolmatage (Detournay).

Les constructeurs d'outil s'attachent d'ailleurs à remédier à cet inconvénient en développant des concepts d'outil qui neutralisent partiellement cet effet défavorable d'extrême sensibilité de l'avance ô au poids W principalement dû à la roche.

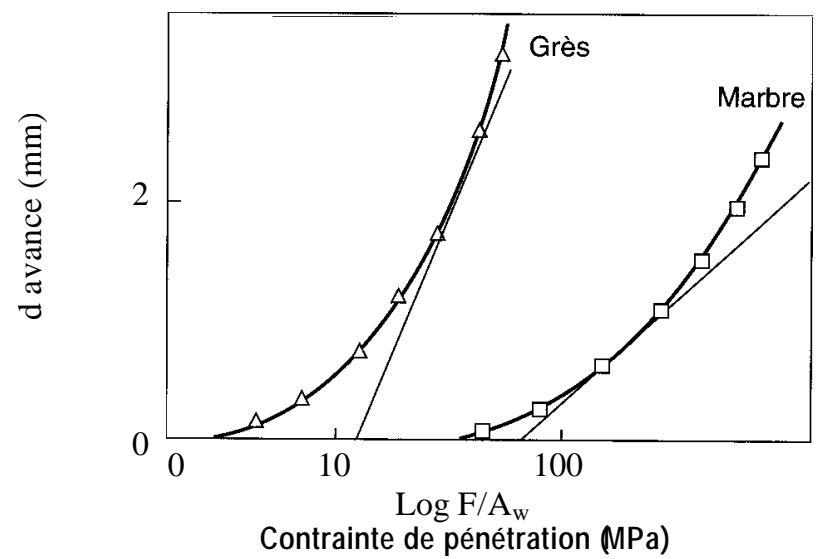

Figure 11

Mise en évidence d'un seuil d'efficacité pour deux types de roche (d'après Glowka, 1985).

Highlighting of an efficiency threshold for two types of rock (according to Glowka, 1985).

\section{Assimilation de la loi de forabilité à une hypothèse de type Archard}

En définitive, et ceci de manière un peu inattendue, la proportionnalité : épaisseur de copeau effort normal, à l'effet de seuil près, est assez conforme à l'hypothèse d'Archard avancée par Théron (1996).

Mais le coefficient de proportionnalité est loin de s'apparenter à une raideur élastique, comme cela a été annoncé dans certaines publications, mais s'apparente plutôt aux propriétés de rupture de la roche ou à une certaine plasticité.

Il est assez connu que les constituants des roches ont un module élastique relativement peu variable (10 à 40 GPa) alors qu'un étagement bien plus important est noté sur les cohésions : une roche tenace a couramment une résistance à la rupture de $100 \mathrm{MPa}$, tandis qu'une roche tendre ou poreuse, présentant des joints de grain défaillants, a une résis tance à la rupture de $5 \mathrm{MPa}$.

Aussi, la résistance opposée à la pénétration de la roche est-elle davantage sous la dépendance des effets de rupture, de désagrégation des joints de grain et non d'un rebond élastique à proprement parler. Il en est déduit que ce n'est pas le module qui intervient mais une grandeur liée à l'énergie spécifique de rupture.

\section{Modèles de poinçon en plasticité}

Dans les tentatives de recoupement avec des modèles, en plasticité, de poinçons de profil soit cylindrique, soit assimilable à un angle, la difficulté (bien identifiée par Detournay (1992), étudiée par Geoffroy (1996) et exploitée par Geoffroy (1997a et b)) pour évaluer l'efficacité, avec l'usure, d'un outil de type PDC, consiste à comptabiliser les parts respectives de la coupe et du frottement occasionné par le méplat d'usure ;1'ambiguité subsiste avec l'outil tricône qui comporte lui aussi une composante cisaillement jointe à la composante poinçonnement. 
Ainsi, un calcul «brutal », en plasticité parfaite, de poinçon cylindrique fait-il apparaître une dépendance linéaire du poids vis-à-vis de la pénétration, tandis que le poinçon pris matique montre une dépendance en $d^{2}$. Un commentaire a posteriori sur cette constatation serait que le poinçon cylindrique réalise, de par sa géométrie ambiguë, un heureux compromis entre l'action de coupe (face inclinée) et celle de frottement (méplat).

\section{Raideur d'outil et raideur de taillant}

En ce qui concerne les valeurs numériques proprement dites entrant dans l'hypothèse de la loi d'Archard : d $=K_{u} F_{N}$, il est important de remarquer que la souplesse de la structure en interaction est notablement plus faible pour l'ensemble de l'outil constitué de $n$ taillants effectivement en contact que pour chacun d'eux pris individuellement.

Comme ordres de grandeur, on pourra considérer $\mathrm{K}_{\mathrm{u}}=10^{-1} \mathrm{~m} / \mathrm{N}$ dans le cas de l'outil global, sur la base d'une avance de $1 \mathrm{~mm}$ pour un effort de $100 \mathrm{kN}$, contre $10^{-6} \mathrm{~m} / \mathrm{N}$ pour un taillant supportant une charge de $1 \mathrm{kN}$ et pénétrant également de $1 \mathrm{~mm}$.

\subsubsection{Raideur dynamique de structure pour le système de forage $(\mathrm{K}$}

En pratique, le diagramme à lobes est plus compliqué que ne l'indique la structure simplifiée présentée au chapitre 2.1, puisque le système présente plusieurs modes significatifs, comportant chacun son propre diagramme à lobes dont il faut assurer la cohérence mutuelle.

Dans ce qui suit, notre intérêt porte désormais sur la raideur dynamique de structure $K_{B}$ dite aussi raideur modale. Cet alinéa vise à vérifier la cohérence des ordres de grandeur déduits de quelques sources d'information complémentaires.

Zamudio (1988), dans une application de forage particulière, effectue un calcul où les valeurs suivantes, portant sur les 3 premiers modes longitudinaux, présentent au moins un intérêt indicatif d'ordre de grandeur

- tiges 4,5" sur $2200 \mathrm{~m}$ précédant $230 \mathrm{~m}$ de masses-tiges 6,5";

- ensemble de suspension de masse environ $10 \mathrm{t}$, avec une raideur de mouflage d'environ $10 \mathrm{t} / \mathrm{cm}$, soit $10^{l} \mathrm{~N} / \mathrm{m}$.

$0,345 \mathrm{~Hz} \quad K_{B} \quad 2,6 \quad 10^{5} \mathrm{~N} / \mathrm{m}$

$1,25 \mathrm{~Hz} \quad K_{B} \quad 1,2 \quad 10^{7} \mathrm{~N} / \mathrm{m}$

$2,29 \mathrm{~Hz} \quad K_{B}=1,0 \quad 10^{8} \mathrm{~N} / \mathrm{m}$

Le premier mode ci-dessus concerne le mode de suspension de la garniture de forage et est associé à une période qui, pour les vitesses de rotation usuelles, ne risque pas de coïncider avec le rythme de défilement des cônes, généralement plus rapide. Le troisième mode est certainement le plus susceptible d'interagir avec le fond.

Par ailleurs, il est instructif d'utiliser les résultats concernant les raideurs modales de la poutre équivalente sollicitée en traction-compression en faisant l'hypothèse simplificatrice due à Dareing (1984), selon laquelle les masses-tiges vibrent en quart d'onde, avec condition de déplacement imposée au fond.

Les résultats généraux suivants sur les forces généralisées $\left(m_{n}\right.$ et $k_{n}$ respectivement masse et raideur généralisée) sont les suivants

$$
k n=m_{n} w_{2 n}
$$

qui, dans le cas de l'exemple précité, se particularisent en ( $\mathrm{S}$ et L désignant respectivement l'aire de la section droite matérielle et la longueur de masses-tiges)

$$
\begin{gathered}
m_{1}=\rho S L \\
m_{n}=\frac{\rho S L}{2} \\
k_{n}=(n-1)^{2} \frac{\Pi^{2}}{2} \frac{E S}{L}
\end{gathered}
$$


et l'on constate que $k_{2}=10^{8} \mathrm{~N} / \mathrm{m}$ est un ordre de grandeur tout à fait réaliste, compatible avec les données précédentes. (Ribes, dans l'étude CEA de 1993, avait adopté $1,4510^{8} \mathrm{~N} / \mathrm{m}$ ).

La fréquence caractéristique des masses-tiges vibrant en quart d'onde est, dans le cas des ondes de compression

$$
N=\frac{1}{4 L} \sqrt{\frac{E}{\rho}}
$$

La valeur numérique associée est voisine de $3 \mathrm{~Hz}$.

En résumé, les différentes théories montrent un accord, au moins qualitatif, très satisfaisant.

\section{COHÉRENCE FORABILITÉ ET DYNAMIQUE POUR L'OUTIL TRICÔNE}

\subsection{Objectif - Description d'un cycle de rebond et hypothèses de travail}

Nous souhaitons proposer une amorce de justification mécanique au décalage (fig. 11) entre poids sur outil et couple observé lors des phases de rebond d'un outil tricône.

Spanos (1994) indique qu'un tel déphasage a déjà été remarqué par Cunningham (1968).

Il est intéressant de considérer un mécanisme de vibration moins intense que le rebond proprement dit. Un exemple d'enregistrement de chantier de ce dernier phénomène est fourni (fig. 13). Les fortes non-linéarités ne nous permettent plus d'appliquer la théorie de Tlusty.

Dans le cas qui retient notre attention, le poids sur outil (WOB ou W) ne paraît pas s'annuler complètement.

Le couple (TOB ou T) lui, semble avoisiner des valeurs non significativement différentes de zéro pendant une proportion de temps relativement importante. Cela est probablement à mettre en relation avec les effets de seuil mentionnés précédemment et peut-être, également, avec des effets complémentaires évoqués plus loin.

Une autre interprétation fait observer que le zéro du capteur de poids n'est pas garanti avec suffisamment de précision. Dans ce qui suit, nous ignorons cette difficulté.

La montée en poids semble précéder celle du couple, ce qui est assimilé à une avance de phase (voir fig. 15 et chapitre 3.3 pour l'interprétation qui en est faite). La décharge se caractérise par une réduction de ce décalage.

Pour simplifier, nous demeurons dans l'hypothèse de linéarité entre l'effort W et l'avance 8 qui est celle de Tlusty. Nous adjoignons à la modélisation Tlusty, qui demeure exclusivement longitudinale, une composante « couple » issue du modèle de tricône de Warren (1984) dont l'objet est de lier le poids W et le couple T. Ce modèle a également été repris en dynamique par Spanos (1994). Ceci nous permet d'utiliser un schéma de représentation mettant en lumière les raisons d'un écart de phase entre couple et poids.

Temps (s)

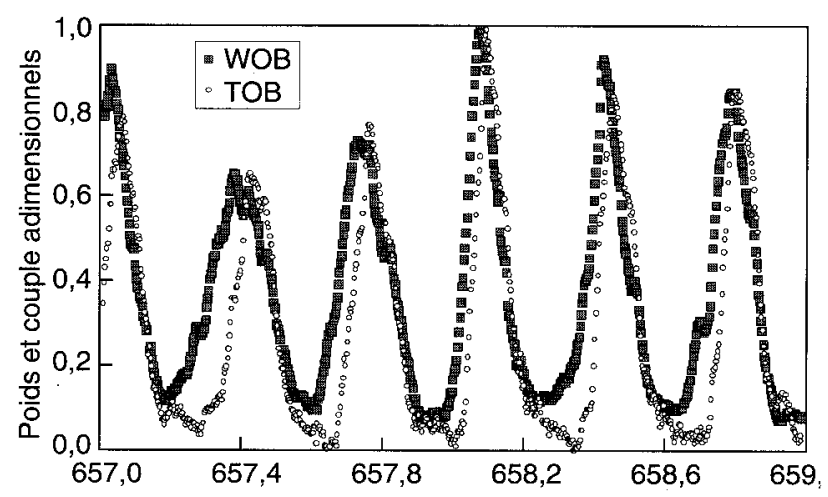

Figure 12

Exemple d'enregistrement temporel du couple (TOB) et du poids (WOB) pour un régime de quasi-rebond de l'outil tricône.

Example of temporal recording of the torque (TOB) and of the weight (WOB) for a near-bouncing speed of the roller cone bit. 

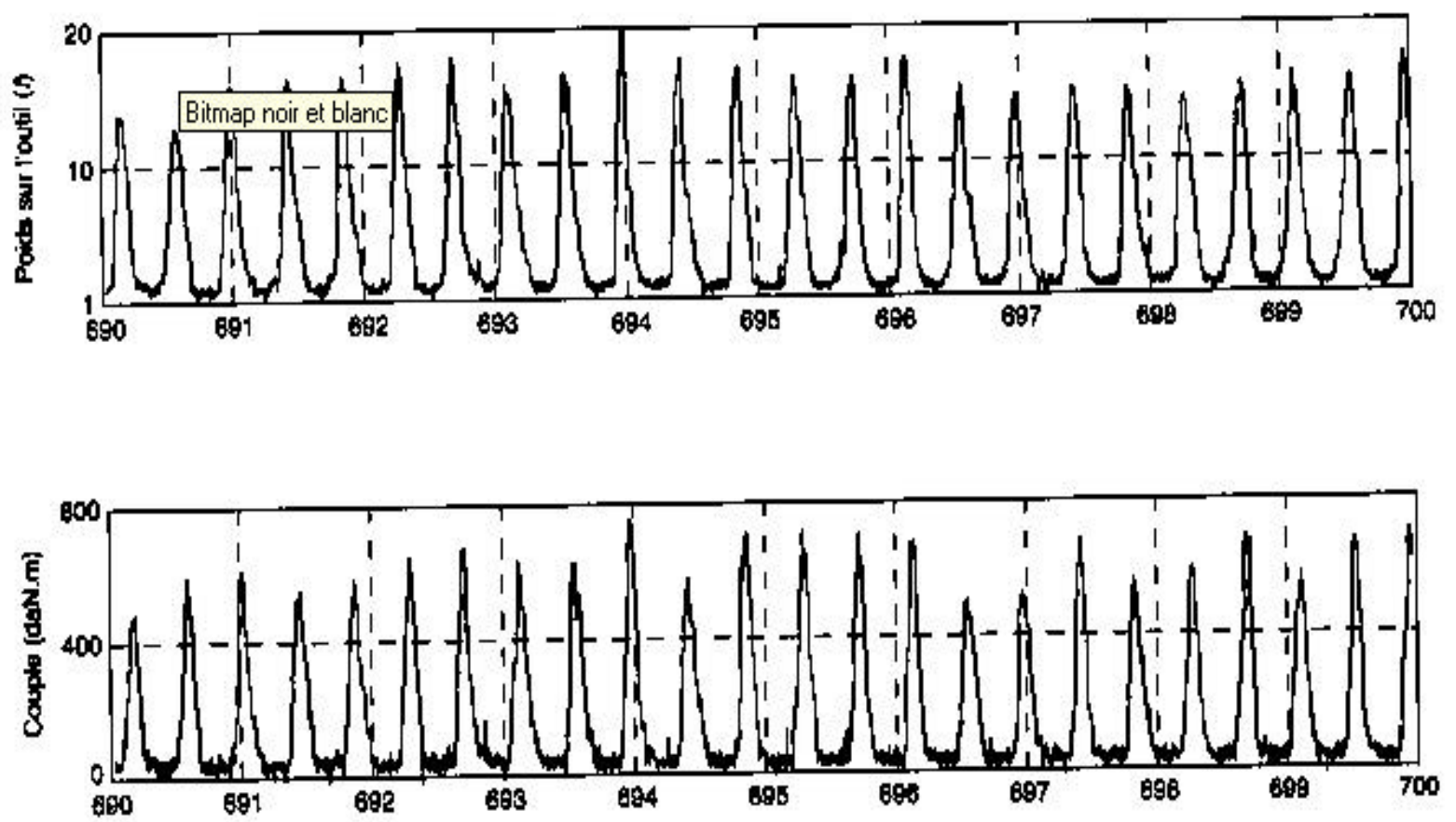

Figure 1.?

Exemple de rebond urès caractọist.

Example of wers chametericed liff-uff dymemicr.

\subsection{Modèle de base de Warren et utilisations dérivées tenant compte de la présence d'untrilobe}

Les modèles mécaniques d'outil de forage s'attachent volontiers à relier entre elles les réponses partielles avance et couple. Warren a présenté une analyse très détaillée qui, résumée en variables de réponse cinématique (longitudinale) et énergétique (circonférentielle), se traduit par la relation affine

$$
T_{D}=c_{1}+c_{2} \sqrt{R_{D}}
$$

avec:

$$
T_{D}=\frac{1}{D} \frac{T}{W} \quad R_{D}=\frac{1}{D} \frac{R}{N} \quad(D \text { désignant le diamètre d'outil })
$$

Elle vaut en conditions de fonctionnement normales, c'està-dire en l'absence de vibrations significatives et pour une roche homogène, présentant des caractéristiques à la rupture bien définies. et et $\mathrm{c}_{2}$ sont des coefficients dépendant à la fois de l'outil et de la roche.

L'ondulation de l'interface induit une perturbation du couple qui modifie sensiblement le schéma précédent. La représentation évoquée vaut pour l'outil tricône. Celle-ci nous intéresse particulièrement, mais il est instructif, pour l'amélioration de la modification suggérée par Spanos (1994) à propos du tricône en condition de formation d'un motif trilobé, de tenir également compte de la discussion de Tlusty (1986) résumée dans ce qui suit.

\subsubsection{Facteur 1 : amortissement longitudinal}

(effet de méplat, sensible pour les outils PDC) (Tlusty, 1986)

Pour Tlusty (1986), le flanc de l'outil d'usinage (assimilable, dans sa position, au méplat d'un taillant PDC) porte de manière plus prononcée sur le massif en position de descente ; l'effort vertical correspondant dit de poids ou normal, est en phase avec la vitesse de progression longitudinale de l'outil et peut, par conséquent, être assimilé à un effet d'amortissement. Tlusty modifie donc la loi de forabilité en jouant, à l'aide d'un amortissement, sur un décalage de phase entre l'effort $F$ et l'épaisseur du copeau $Z-Z_{0}$. Un modèle dans ce sens a été examiné par Mazelet (1995).

\subsubsection{Facteur 2 : modification du rapport effort vertical/effort horizontal pour la géométrie tricône (Spanos, 1994)}

Spanos remanie le modèle de Warren et s'intéresse à la modification de la relation citée existant entre poids et couple à l'outil, compte tenu de l'ondulation d'interface (ceci se traduit par une perturbation sur $T_{D}$ ). La position de descente induit, au niveau du roulement du cône, un allégement relatif de l'effort horizontal de coupe contribuant au couple, ce qui est bien intuitif.

C Putot et C Mabile /Conditions d'apparition et formes de rebond d'un outil de forage tricône 


\subsubsection{Réduction de la pression et de la pénétration des taillants par le biais de situations de conformité géométrique du cône et de l'interface}

Notre idée est qu'au terme précédent (facteur 2) vient s'ajouter, pour les tricônes, un terme géométrique de même nature que celui mentionné par Tlusty pour les outils d'usinage, bien que pour la géométrie tricône il s'exprime différemment.

Dans le cas d'une interface plane associée à un tricône (fig. 14a), un poids sur outil important fait participer davantage de dents en redistribuant les efforts, de sorte que les schémas de forabilité usuels, fondés sur l'hypothèse de pénétration d'une seule dent, rien rendent pas compte.

De la même manière (fig. 146), dans le cas d'interfaces compatibles (creux du trilobé), une pénalité sur la vitesse de pénétration instantanée de l'outil dans la formation (rate of penetration ou ROP) devrait être constatée dans la phase découpe des « creux », associée à une perte d'efficacité, d'autant plus que l'amplitude des bosses du trilobé est importante. À l'inverse, sur les parties saillantes, l'effort reste concentré sur un nombre de dents limité.

\subsubsection{Choix effectué}

Les modifications de performance traduites par $T_{D}$, notre critère d'efficacité, seront prises dans ce travail sur la base des facteurs 2 et 3

- facteur $2: T_{D}$ est faible dans les descentes des ondulations, en raison de la diminution de l'effort horizontal s'exerçant sur les cônes.

a
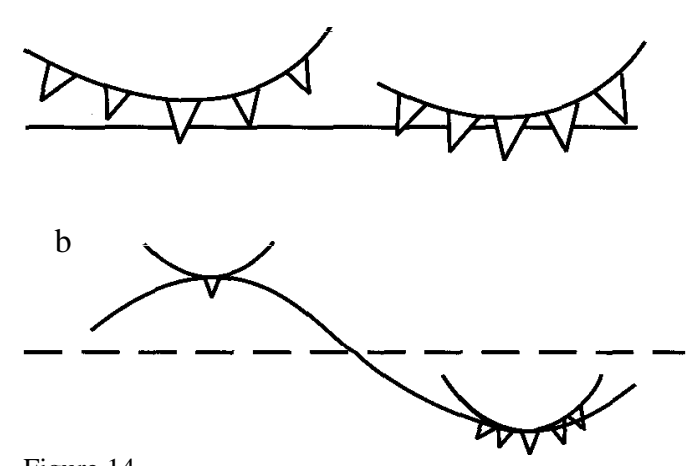

Figure 14

Efficacité de coupe et conformité de surfaces. a : lorsque le poids sur outil s'accroît, il y a redistribution d'effort. b : la plus ou moins bonne compatibilité géométrique des surfaces induit des effets comparables à ceux visés en a.

Cutting efficiency and surface conventionality. a: when thé weight on the bit increases, there is a redistribution of load. b: thé more or less correct geometrical compatibility of the surfaces induces effects which are comparable to those observed in a.

\section{Modulation d'épaisseur du copeau}

$\mathrm{t}$

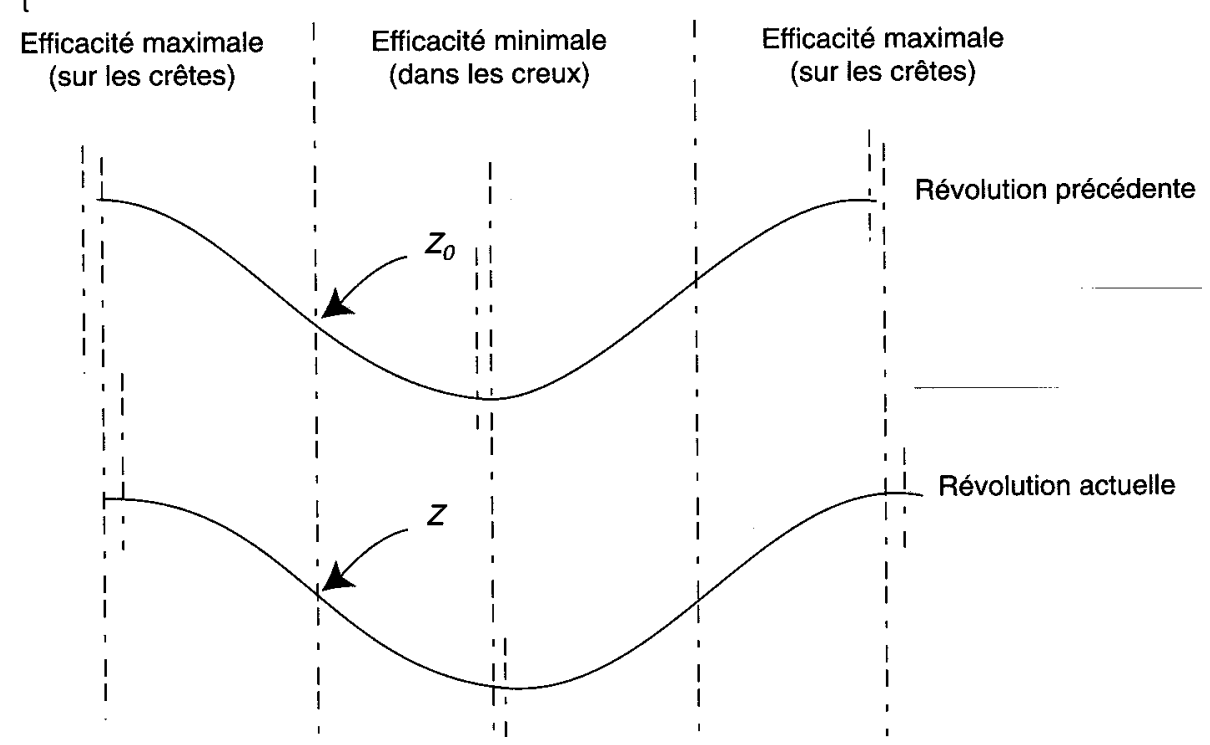



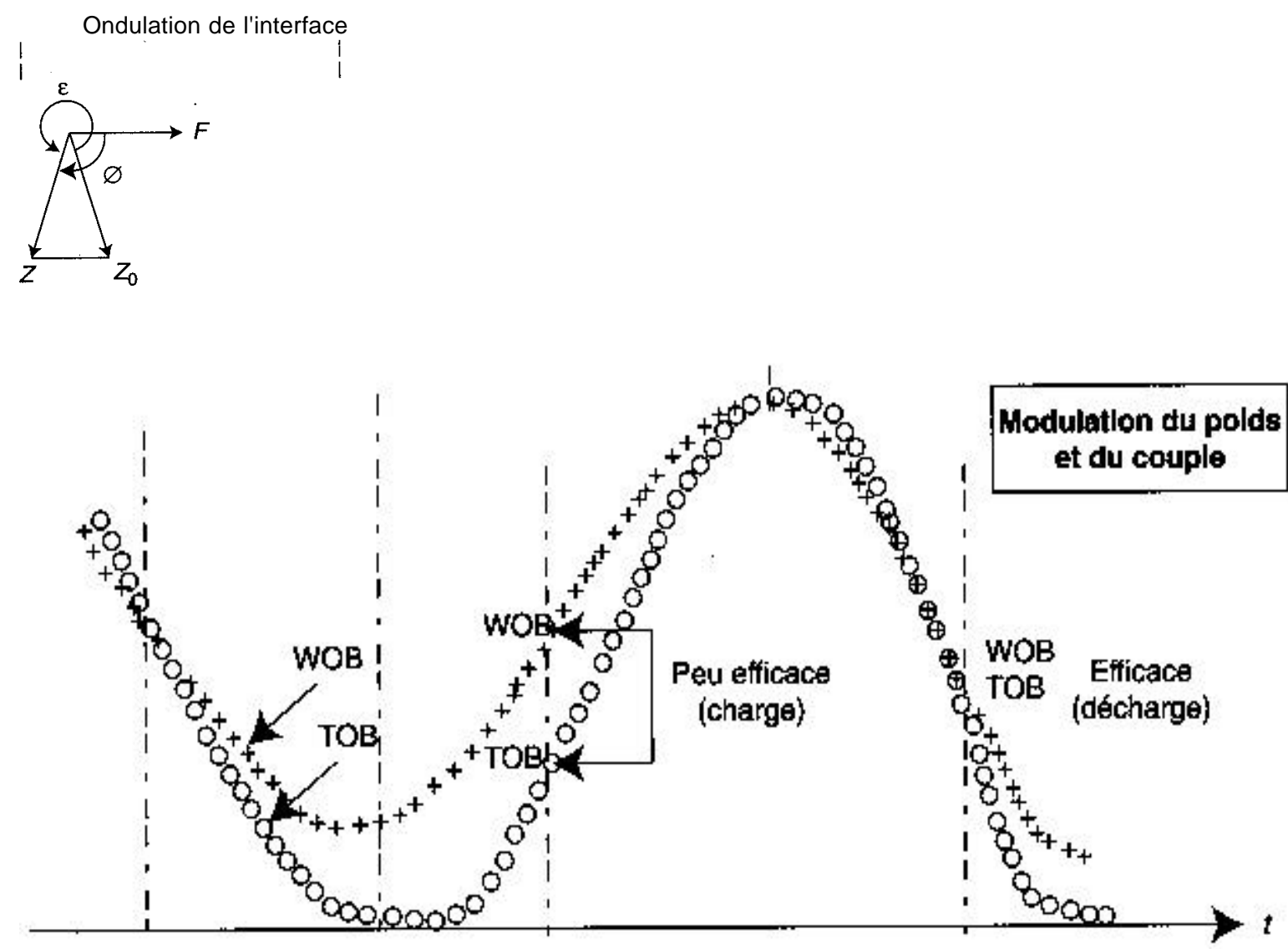

Figure 15

Phase des différentes grandeurs caractérisant le comportement de l'interface : poids, couple, cote du trilobe. Phase of the diffèrent magnitudes characterising the behavior of the interface: weight, torque, value of the lobe. 


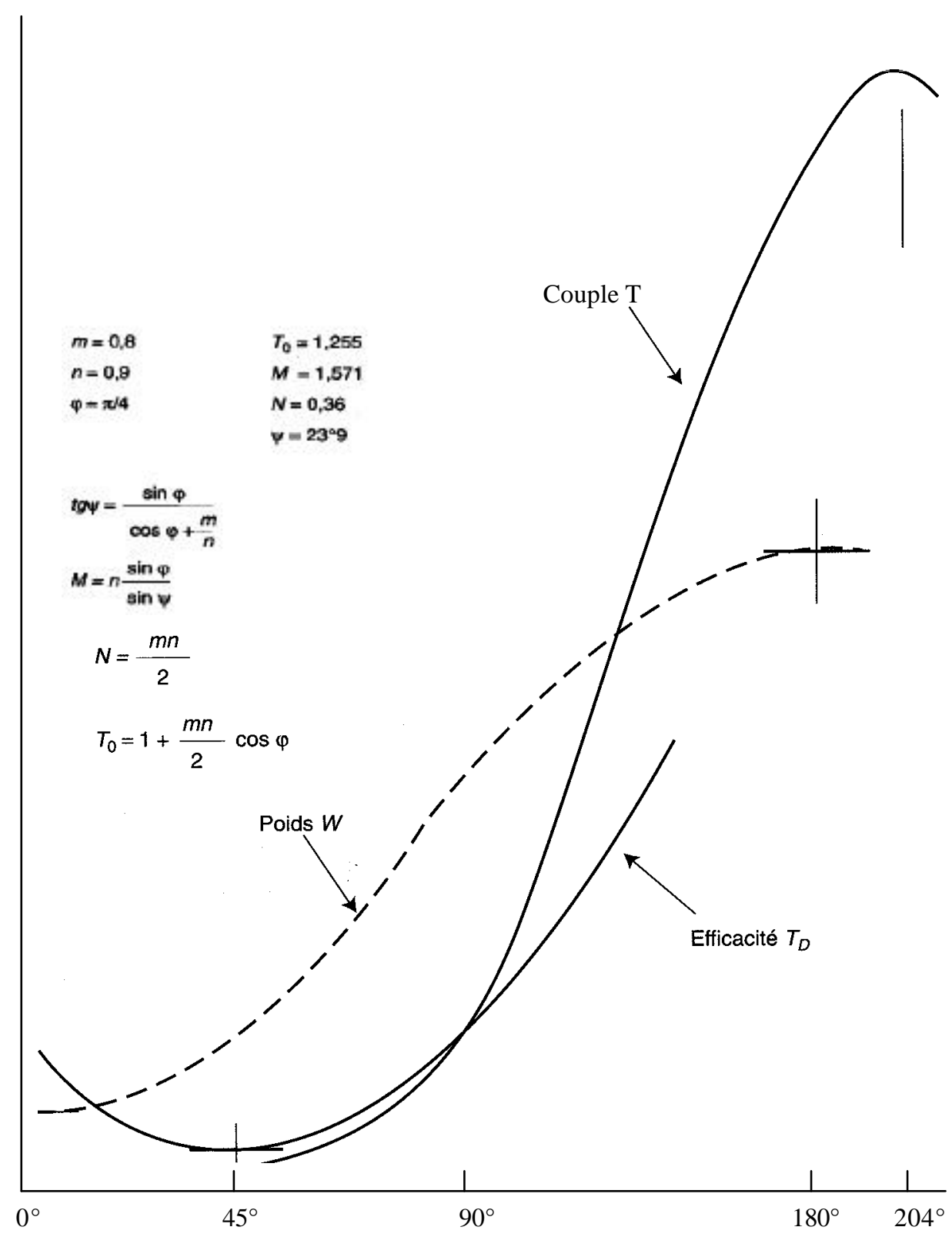

Minimum du

Creux du trilobe

copeau

\section{Figure 16}

Illustration numérique d'un modèle de représentation des décalages de phase entre poids, couple et efficacité ; mise en évidence d'un facteur de forme sur le couple au voisinage des extrema.

Numerical illustration of a representative model of the phase shifts between weight, torque and efficiency; highlighting a factor of shape on the torque around the extreme limits.

- facteur $3: T_{D}$ est faible dans les creux, car il y a redistribution des efforts en raison d'une compatibilité d'interface.

La conjugaison de ces deux facteurs, supposés intervenir simultanément, invite à présumer que le minimum d'efficacité se situe entre le minimum tranché $\mathrm{S}=\mathrm{Z}-\mathrm{Z}_{\mathrm{o}}$ (donc de poids $\mathrm{W}$ ) et le creux du trilobe. Comme on le verra par la suite, cette hypothèse naturelle cadre parfaitement avec l'allure des enregistrements

- sur le plan des décalages de phase entre poids W et couple T ;

- sur la forme même des enregistrements temporels de ces grandeurs.

\subsection{Utilisation des résultats de l'analyse Tlusty}

Il reste à exploiter (fig. 15) le diagramme de Nyquist présenté par Tlusty (fig. 8a), particularisé au problème d'entretien d'ondulations, avec notamment les positions des vecteurs «tournants »: effort de poids $F$, ondulation $\mathrm{Z}$ ou $\mathrm{Z}_{\mathrm{o}}$, copeau $\mathrm{Z}-\mathrm{Z}_{\mathrm{o}}$. L'inconnue réside dans le décalage effectif $\mathrm{E}$; si l'on admet un faible déphasage entre 
deux passages successifs ( $E$ un peu inférieur à $2 n$ ), l'effort vertical et donc l'épaisseur tranchée sont en avance de phase d'environ $\mathrm{n} / 2$ par rapport à l'ondulation $\mathrm{Z}\left(\mathrm{ou} \mathrm{Z}_{\mathrm{o}}\right)$.

Si, parallèlement, on tient compte de la phase supposée de TD, conforme à l'hypothèse vraisemblable exposée au paragraphe précédent, les différents éléments sont en place (fig. 16). Par ordre chronologique de phase, nous avons

- poids W, dont la phase de référence est conventionnellement nulle ; c'est également la phase de l'épaisseur du copeau ;

- efficacité $T D$, en retard de $c p=n / 4$ pour fixer les idées (à mi chemin entre le minimum du copeau et le minimum du trilobé) ; pour le couple $\mathrm{T}$, se reporter au commentaire qui suit ;

- ondulation $Z_{\mathrm{o}}$ laissée au tour précédent, en retard d'environ it/2 (un peu moins) sur le poids ;

- ondulation $\mathrm{Z}$ actuelle, en retard d'environ $\mathrm{n} / 2$ (un peu plus) sur le poids.

Par ailleurs, en ce qui concerne l'allure de la forme des caractéristiques temporelles du couple $\mathrm{T}$ et du poids W nous avons souhaité tirer parti du modèle de Warren (1984) enrichi de la manière dont il vient d'être fait état pour justifier notamment le plateau inférieur de couple $\mathrm{T}$, dont l'existence ne serait pas due exclusivement à des considérations de seuil. La même étude justifie que les crêtes de couple T sont plus pointues que ne sont les creux.

Une simple application numérique (fig. 16) suffit pour s'en convaincre. Nous avons supposé une allure sinusoïdale pour le poids $\mathrm{W}$ et l'efficacité $\mathrm{T}_{D}$, de ce fait, le couple $\mathrm{T}$ comporte un harmonique de fréquence double qui vient se superposer à l'harmonique fondamental en étalant le minimum et effilant le maximum, ce qui est l'effet observé sur les enregistrements Trafor (fig. 13).

Un examen des résultats (fig. 6) obtenus par le CEA (Politopoulos, 1993 ; Ribes, 1993) en calcul non linéaire avec phase de rebond confirme la position intuitive des séquences de vol : décollement du cône au voisinage des crêtes et reprise du contact au voisinage des creux. Cette position est compatible avec la concordance de phase entre le minimum de poids et le milieu de la séquence de descente sur le flanc du lobe.

Par ailleurs, on suppose implicitement que la reprise de la formation par trois cônes identiques se fait selon un mode strictement périodique, ce qui n'est qu'une hypothèse approximative. On sait en fait que la symétrie ternaire n'est pas particulièrement recherchée puisqu'elle induit, précisément, des vibrations. Le plan présenté par Warren (fig. 17) est un exemple d'illustration de variabilité intercônes avec un souhait de briser la symétrie.

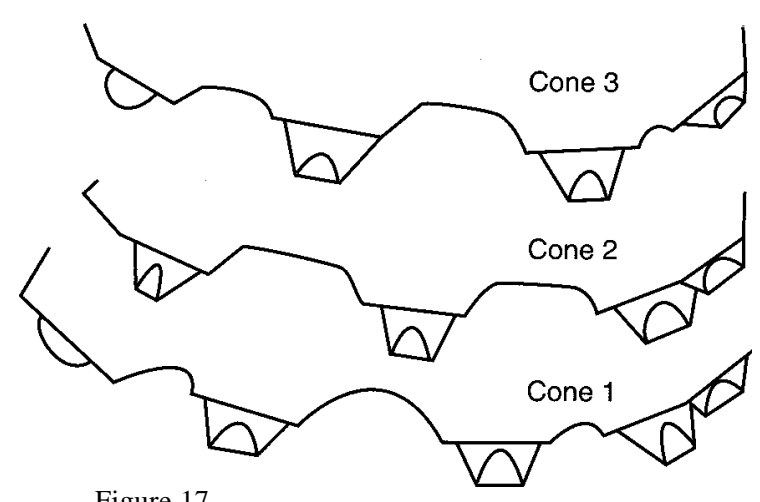

Organisation typique des couronnes de coupe sur les cônes montrant la dissymétrie des trois cônes (d'après Warren, 1984).

Typical organization of thé cutting rows on thé cones showing thé dissymmetry of thé three cones (according to Warren, 1984).

\section{CONCLUSION}

Un modèle classique linéarisé d'autoexcitation conçu pour les vibrations autoentretenues d'une machine d'usinage des métaux - modèle dû à Tlusty (1986) - a été transposé aux vibrations longitudinales d'un système de forage et extrapolé au rebond. Ce modèle permet de justifier l'existence d'un décalage de phase entre poids sur outil et couple.

Ce même modèle, complété par la théorie mécanique du tricône de Warren (1984), apporte une explication au manque de symétrie observé dans les enregistrements au voisinage des extrema : plateau du minimum de couple et accentuation du maximum. 
Le modèle de Tlusty montre le lien entre le déphasage entre deux ondulations consécutives et l'amortissement du système ; le déphasage est évidemment à relier à la rotation du motif constaté à chaque révolution de l'outil. Cet élément expérimental peut aider à la détermination de l'amortissement.

Les limites de l'approche se situent évidemment au niveau du choix très rudimentaire de la fonction de transfert du système de forage, mais il n'y a pas d'obstacle de principe à l'augmentation du nombre de degrés de liberté de la représentation associée.

\section{REMERCIEMENTS}

Nous tenons à remercier Isabelle Rey-Fabret, Jean Guesnon et Philippe Perreau pour leur contribution au choix des relevés expérimentaux, la relecture du manuscrit et les discussions et suggestions d'extension de la théorie aux conditions de transition d'un trilobe à un motif d'ordre de symétrie supérieur.

RÉFÉRENCES

Abbassian, F. (1994) Drillstring Vibration Primer.

Aldred, W.D. et Sheppard, M.C. (1992) Drillstring Vibrations: a New Generation Mechanism and Control Strategies. SPE 24582, 67thAnnual Technical Conférence, Washington D.C.

Cooley, C.H., Pastusek, P.E. and Sinor, L.A. (1992) Development of an Anti-Whirl Cote Bit. SPE 24586, 67th Annual Technical Conférence and Exhibition, Washington D.C.

Cunningham, R.A. (1968) Analysis of Downholé Measurements of Drill String Forces and Motions. Trans. of ASME, Journal of Engineering for Industry, May, 208-216.

Dareing, D.W. (1984) Drill Collar Length is a Major Factor in Vibration Control. Journal of Petroleum Technology.

Detoumay, E. et Defoumy, P. (1992) A Phenomenological Model for thé Drilling Action of Drag Bits. Int. J. Rock Mech.

Min. Sci. and Geomech.Abstr., 29, 1, 13-23.

Dunayevsky, V.A., Judzis, A. et Mills, W.H. (1985) Dynamic Stability of Drillstrings under Fluctuating Weights on Bit. SPE 14329.

Dunayevsky, V.A. et Abassian, F. (1995) Application of Stability Approach to Bit Dynamics. SPE 30478.

Falconer, I.G., Burgess, T.M. et Sheppard, M.C. (1988) Separating Bit and Lithology Effects from Drilling Mechanics Data. IADC/SPE Drilling Conférence, Dallas.

Geoffroy, H. (1996) Étude de l'interaction roche/outil de forage influence de l'usure sur les paramètres de coupe. Thèse. Geoffroy, H., Nguyen Minh, D. et Putot, C. (1997) Study on Interaction Between Rocks and Worn PDC's Cutter. 36th

Symposium US, New York, Int. J. Rock Mech. \& Min. Sci., 34, 611.

C Putot et C Mabile / Conditions d'apparition et formes de rebond d'un outil de forage tricône 335

Geoffroy, H., Nguyen Minh, D. et Putot, C.-(1997) Rock-PDC Bit Interaction: Study of thé Frictional Contact Between Rock and Cutter Wear Flat. Symposium International, Engineering Mechanics Today 97.

Glowka, D.A. (1985) Implications of Thermal Wear Phenomena for PDC Bit Design and Operation. 60th Annual

Technical Conférence and Exhibition of thé SPE, Las Vegas.

Mabile, C., Desplans, J.P. et Pavone, D. (1996) A New Way of Using Surface Measurements to Detect Down Hole Vibrations. Europec Milan SPE 36883.

Mazelet, A. (1995) Étude des vibrations auto-excitées d'un outil de forage. Mémoire de stage d'élève de l'École polytechnique.

Politopoulos, 1. (1993) Méthodes de calcul du comportement dynamique non linéaire d'une garniture de forage. Rapport CEADMT / 93.212

Putot, C. (1993) Modèles du processus de foration. Rapport interne 40476.

Putot, C. et Constantinescu, A. (1997) Modèle de foration fondé sur le couplage des effets d'évacuation des déblais et de coupe de la roche. Symposium Saint-Venant, ENPC, Paris.

Putot, C., Perreau, P. et Constantinescu, A. (1998) Field Data vs Theoretical Model to Quantify Drilling Efficiency and Disruption. SPE 50579 European Petroleum Conférence, La Haye.

Ribes, F. (1993) Comportement dynamique d'une ligne de forage pétrolier. Rapport de stage ENSTA. Rapport CEADMT/93.381.

Sinor, L.A., Warren, T.M., Behr, S.M. et Wells, M.R. (1992) Development of an Anti-Whirl Core Bit. SPE 24587, 67th

Annual Technical Conférence and Exhibition, Washington D.C.

Spanos, P.D., Sengupta, A.K., Cunningham, R.A. et Paslay, P.R. (1994) Modeling of Roller Cone Bit Lift-Off Dynamics in Rotary Drilling. Drilling Technology ASME, 56.

Théron, A. (1996) Modélisation des effets non linéaires de contact dans le processus de forage. Thèse.

Tlusty, J. (1986) Dynamics of High-Speed Milling. Journal of Engineering for Industry, 108, 59-67.

Tobias, S.A. (1961) Machine-Tool Vibration. Shock and Vibration Handbook, Mc Graw Hill.

Warren, T.M. (1984) Factors Affecting Torque for a Roller Cone Bit. Journal of Petroleum Technology, 1500-1508.

Winters,W.J., Warren, T.M. et Onyia, E.C. (1987) Roller Bit Model with Rock Ductility and Cone Offset. 62nd Annual Technical Conférence and Exhibition of thé SPE, Dallas.

Wu, D.W. (1989) A New Approach of Formulating thé Transfer Function for Dynamic Cutting Processes. Journal of Engineering for Industry, 111, 37.

Zamudio, C.A., Tlusty, J.L. et Dareing, D.W. (1987) Self-Excited Vibrations in Drillstrings. SPE 16661.

Zamudio,C.A., Tlusty, J.L. et Dareing, D.W. (1988) Effect of Shock Absorber on Drag Bit Chatter. SPE 17194. 